Mens

Revue d'histoire intellectuelle et culturelle

mens

\title{
La pensée nationaliste de Denis-Benjamin Viger (1809-1837)
}

\section{Martin Lavallée}

Volume 14, numéro 1, automne 2013

URI : https://id.erudit.org/iderudit/1032622ar

DOI : https://doi.org/10.7202/1032622ar

Aller au sommaire du numéro

Éditeur(s)

Centre de recherche en civilisation canadienne-française

ISSN

1492-8647 (imprimé)

1927-9299 (numérique)

Découvrir la revue

Citer cet article

Lavallée, M. (2013). La pensée nationaliste de Denis-Benjamin Viger (1809-1837). Mens, 14(1), 41-89. https://doi.org/10.7202/1032622ar

\section{Résumé de l'article}

Denis-Benjamin Viger (1774-1861) est l'un des principaux chefs de file du mouvement patriote bas-canadien jusqu'à la rébellion de 1837 . Très actif politiquement, Viger est également l'auteur d'importants ouvrages politiques qui s'inscrivent dans la foulée des revendications canadiennes à l'égard des autorités coloniales britanniques. Pourtant, malgré la présence de ces sources importantes qui en disent long sur ses motivations et sur ses idées et malgré le rôle prépondérant qu'il a joué au cours de cette période mouvementée du Bas-Canada, les historiens ont peu étudié le personnage et sa pensée particulière, sur lesquels la lumière reste à faire. Ainsi, cet article analyse le discours et les actions politiques de Viger de 1809 jusqu'à la veille de la rébellion de 1837. L'examen de ses principaux écrits nous amène à saisir la logique de la pensée de ce chef patriote. Nous mettons ainsi en relief sa pensée nationaliste, pensée qui constitue le moteur de ses actions et revendications durant toute la période à l'étude. Par le fait même, nous contribuons à remettre à l'avant-plan cette dimension nationale dans l'historiographie intellectuelle des rébellions au Bas-Canada. 


\title{
La pensée nationaliste de Denis-Benjamin Viger $(1809-1837)^{1}$
}

\author{
Martin Lavallée \\ Université du Québec à Montréal
}

\section{Résumé}

Denis-Benjamin Viger (1774-1861) est l'un des principaux chefs de file du mouvement patriote bas-canadien jusqu’à la rébellion de 1837. Très actif politiquement, Viger est également l'auteur d'importants ouvrages politiques qui s'inscrivent dans la foulée des revendications canadiennes à l'égard des autorités coloniales britanniques. Pourtant, malgré la présence de ces sources importantes qui en disent long sur ses motivations et sur ses idées et malgré le rôle prépondérant qu'il a joué au cours de cette période mouvementée du Bas-Canada, les historiens ont peu étudié le personnage et sa pensée particulière, sur lesquels la lumière reste à faire. Ainsi, cet article analyse le discours et les actions politiques de Viger de 1809 jusqu'à la veille de la rébellion de 1837. L'examen de ses principaux écrits nous amène à saisir la logique de la pensée de ce chef patriote. Nous mettons ainsi en relief sa pensée nationaliste, pensée qui constitue le moteur de ses actions et revendications durant toute la période à l'étude. Par le fait même, nous contribuons à remettre à l'avant-plan cette dimension nationale dans l'historiographie intellectuelle des rébellions au Bas-Canada.

\footnotetext{
${ }^{1}$ Je remercie madame Fernande Roy, qui m'a guidé dans la rédaction du mémoire de maittrise à l'origine de cet article. Merci également aux évaluateurs anonymes pour leurs commentaires et conseils judicieux qui ont permis d'améliorer cet article.
} 


\section{Abstract}

This article is part of a larger study which focuses on the thought and political actions of Denis-Benjamin Viger during the first decade of the union of the Canadas (1840-1850). In preparing this study, however, it was necessary to examine the period that preceded the union and the Lower Canadian Rebellions of 1837-1838. In so doing, we have come to better understand the ideas that fed Viger's thought, many of which have received scant attention in the scholarly literature on the rebellions. Recent historiography has focused on the republicanism and pro-Americanism of the rebel leadership, as well as their adherence to enlightenment philosophy. However, little attention has been devoted to the other currents of thought that influenced some rebel leaders, which has led historians to overly homogenise the Patriot thought. Moreover, contemporary scholarship tends to ignore nationalism as a motivating factor for Patriot agitation in the 1830s. This article seeks instead to shed new light on the variety of ideas that motivated one of Lower Canada's leading political figures, Denis-Benjamin Viger, to take part in the radicalisation of Patriot discourse from 1834 to 1837. In so doing, it will show that the Patriot movement of the 1830s was not a homogeneous ideological bloc and that a number of motivations fed the discontent of Patriot leaders towards British colonial power. 
Le dernier acte qui a finalement réglé la forme de notre gouvernement et qui nous assure la jouissance de nos droits comme sujets Britanniques, avoit pour but avoué de consolider en même temps ceux qui nous appartenoient, [...] en nous en confiant le dépôt. C'étoit pour nous mettre à même de conserver nos loix, nos usages, notre langue, notre culte, que l'on a donné au Bas-Canada, en particulier un gouvernement séparé. Denis-Benjamin Viger ${ }^{2}$

Dans cet énoncé, nous tenons le fondement de la pensée de Viger, celle qui le guidera dans ses actions durant toute sa carrière politique. Ces deux phrases résument la conception que se faisait Viger du Bas-Canada et de la nation canadienne avant les rébellions de 18371838, et qu'il cherchera à appliquer après l'Union de 1840. Cette interprétation de l'Acte constitutionnel de 1791 est également la source d'un malentendu qui ne sera résolu qu'après les rébellions de 1837-1838, lorsque la population canadienne sera mise en minorité au sein d'une union législative dont l'objectif avoué est de la noyer au sein de la majorité britannique. Car, jusqu'en 1840, la vision de Viger concernant le Bas-Canada, la nation canadienne et l'Acte de 1791 est confrontée à celle d'une oligarchie coloniale britannique qui conçoit le Bas-Canada, avant tout, comme une colonie à vocation commerciale et dont la population est appelée, à terme, à devenir entièrement britannique.

Denis-Benjamin Viger (1774-1861) est l'un des intellectuels et hommes politiques les plus importants du premier tiers du XIX ${ }^{\mathrm{e}}$ siècle canadien. Après la chefferie de Pierre-Stanislas Bédard, Viger est, avec son cousin Louis-Joseph Papineau, l'un des principaux chefs de file canadiens jusqu'à la rébellion de l'automne de 1837 . Toutefois, contrairement à ces deux grands politiciens canadiens sur lesquels

\footnotetext{
${ }^{2}$ Denis-Benjamin Viger, Analyse d'un entretien sur la conservation des etablissemens du Bas-Canada, des loix, des usages, \& c de ses habitans, Montréal, Imprimé chez James Lane, 1826, p. 31, [En ligne], [http://collections.banq.qc.ca/ bitstream/52327/2022363/1/160359.pdf].
} 
plusieurs études se sont penchées ${ }^{3}$, Viger a peu retenu l'attention des historiens jusqu'ici et il faut remonter aux années 1970 pour trouver une étude scientifique consacrée à un aspect de sa carrièré ${ }^{4}$.

Nous croyons que cette lacune de l'historiographie est attribuable au fait que les influences idéologiques de Viger sont diverses et que ce dernier est un personnage difficile à cerner et qui peut même paraître parfois contradictoire. En effet, dans les différents ouvrages portant sur la période précédant les rébellions dans lesquels Viger est mentionné, certains de ses écrits sont tantôt apparentés au républicanisme $e^{5}$, tantôt au conservatisme ${ }^{6}$, en plus du fait que des chercheurs voient en lui un porteur du principe des nationalités ${ }^{7}$ et que d'autres soutiennent, par ailleurs, que sa pensée n'est pas nationaliste ${ }^{8}$. En outre, après l'Union des Canadas, Viger a soulevé la perplexité de plusieurs historiens pour s'être dissocié des réformistes de LouisHippolyte La Fontaine et pour avoir accepté de siéger avec les tories

3 En ne tenant compte que des études récentes, voir notamment le dossier consacré à " Pierre-Stanislas Bédard, la crise de 1810 et les débuts de la démocratie parlementaire ", dans le Bulletin d'histoire politique, vol. 19, nº 3 (printemps 2011); voir également Gilles Gallichan, "Pierre Bédard et les patriotes de 1810 », dans Charles-Philippe Courtois et Julie Guyot (dir.), La culture des patriotes, Québec, Éditions du Septentrion, 2012, p. 43-58. Pour Papineau, voir, entre autres, les travaux d'Yvan Lamonde, « Britannisme et américanité de Louis-Joseph Papineau à l'époque du deuxième projet d'Union (1822-1823) ", Les Cahiers des Dix, n 66 (2012), p. 55-94; Yvan Lamonde et Jonathan Livernois, Papineau : erreur sur la personne, Montréal, Éditions du Boréal, 2012; voir également Gilles Laporte, "Louis-Joseph Papineau et les enjeux locaux à la veille de la rébellion de 1837 ", dans Courtois et Guyot (dir.), La culture des patriotes, p. 119-140.

${ }^{4}$ André Lefort, Les deux missions de Denis-Benjamin Viger en Angleterre, en 1828 et de 1831 à 1834, thèse de doctorat, Montréal, Université McGill, 1975; voir également l'ouvrage de vulgarisation de Gérard Parizeau, La vie studieuse et obstinée de Denis-Benjamin Viger, Montréal, Éditions Fides, 1980.

5 Voir Marc Chevrier et al., De la République en Amérique française, Québec, Éditions du Septentrion, 2013.

${ }^{6}$ Michel Ducharme, Le concept de liberté au Canada à l'époque des révolutions atlantiques, Montréal, McGill-Queen's University Press, 2010, p. 281-282.

7 Yvan Lamonde, Histoire sociale des idées au Québec, t. 1 : 1760-1896, Montréal, Éditions Fides, 2000, p. 223.

8 Ducharme, Le concept de liberté au Canada. 
du Haut-Canada au Conseil exécutif du gouverneur Charles Metcalfe. Viger se range donc difficilement dans une catégorie idéologique spécifique et c'est la raison pour laquelle, selon nous, l'homme n'a pas fait l'objet d'études approfondies de la part des chercheurs. Nous allons tenter de pallier cette lacune, en nous limitant toutefois à la période qui précède les soulèvements de 1837-1838 .

La thèse défendue dans cet article est que l'élément déterminant de la pensée de Viger, et qui lui donne tout son sens, est son nationalisme, que nous définissons comme ce "sentiment national fort de la part d'un individu, d'un groupe ou d'une population ${ }^{10}$ " et qui pousse Viger à se porter à la défense de ce qu'il considère être sa nation face aux menaces auxquelles elle est confrontée. Nous avançons donc que la cohérence et la portée des écrits et des actions de Viger, du début du XIX ${ }^{e}$ siècle jusqu'à l'aube des rébellions de 1837-1838, résident dans le fait que celui-ci cherche énergiquement à conserver l'existence et l'autonomie d'un peuple d'origine, de culture, de langue et de génie français sur le territoire du Bas-Canada. Nous verrons également que cette pensée nationaliste s'allie à une pensée conservatrice critique du libéralisme marchand et du progressisme libéral des Lumières, qui entend faire table rase des spécificités historiques et culturelles des sociétés au nom d'un universalisme abstrait incarné par les droits de l'homme. Si Viger est loin d'être opposé à ce que les individus possèdent des droits, il refuse toutefois que ces droits se superposent au droit des sociétés historiquement constituées, en particulier la nation canadienne.

Cet article va donc contribuer à nuancer certaines interprétations qui ont cours dans l'historiographie des rébellions qui, bien malgré elle, donne parfois l'impression que le mouvement patriote constituait

9 Pour en savoir plus sur Viger après 1840 , nous renvoyons à notre mémoire de maîtrise, Faire échec à l'Union : Denis-Benjamin Viger, un patriote face au CanadaUni, Université du Québec à Montréal, 2013.

${ }^{10}$ Guy Hermet, « Nationalisme », dans Sylvie Mesure et Patrick Savidan (dir.), Le dictionnaire des sciences humaines, Paris, Presses universitaires de France, 2006, p. 801. 
un bloc idéologique homogène ${ }^{11}$. D'un point de vue strictement idéologique, l'historiographie récente s'est particulièrement intéressée au libéralisme et au républicanisme ${ }^{12}$ présents au sein du mouvement, négligeant parfois la dimension nationale des revendications patriotes ${ }^{13}$. En comparant le mouvement patriote bas-canadien à des mouvements similaires ailleurs dans le monde atlantique ou au HautCanada, on constate que la situation particulière de la population bas-canadienne, soumise à la domination d'une puissance étrangère, a été quelque peu mise de côté. De ce fait, la dimension nationale du conflit bas-canadien, considérée essentielle par l'historiographie de la première moitié du $\mathrm{xx}^{\mathrm{e}}$ siècle, a été occultée. Par l'étude de la pensée de Viger, nous replaçons cette dimension nationale au premier plan. De plus, en retraçant le parcours individuel d'un des principaux protagonistes du mouvement patriote, nous montrons implicitement que sous l'apparent consensus du mouvement se dissimulaient, en fait, plusieurs motivations et sensibilités disctinctes les unes des autres.

11 Pour un portrait complet de l'historiographie des rébellions, voir Mathieu Arsenault, L'historiographie des rébellions de 1837-1838 au XX' siècle : débats et rôle structurant dans la construction des grandes représentations de l'histoire du Québec, mémoire de maîtrise, Université du Québec à Trois-Rivières, 2013. Après avoir dominé dans l'historiographie durant toute la première moitié du $\mathrm{xx}^{\mathrm{e}}$ siècle, l'interprétation nationale du conflit patriote a été progressivement négligée au profit des idéaux partagés par l'ensemble des sociétés occidentales du monde atlantique, comme le libéralisme et le républicanisme. Arsenault montre bien comment les diverses interprétations idéologiques des rébellions de la part des historiens contribuent, "sciemment ou non ", à légitimer un projet de société particulier lié à l'idéologie mise de l'avant. Cette analyse d'Arsenault peut, en partie, expliquer l'absence d'études récentes sur Viger, ce dernier entrant difficilement dans les catégories idéologiques privilégiées par l'historiographie récente.

12 Voir les deux ouvrages les plus représentatifs de ces deux courants : pour l'historiographie libérale, Yvan Lamonde, Histoire sociale des idées au Québec; pour l'historiographie républicaine, Louis-Georges Harvey, Le printemps de l'Amérique française : américanité, anticolonialisme et républicanisme dans le discours politique québécois, 1805-1837, Montréal, Éditions du Boréal, 2005.

13 Pour Michel Ducharme, la dimension nationale était tout simplement absente du mouvement bas-canadien (voir Ducharme, Le concept de liberté au Canada, p. 156). 
Nous donnons ainsi raison à certains historiens qui posaient comme hypothèse que le mouvement patriote n'était pas monolithique ${ }^{14}$.

Pour saisir le mieux possible la pensée singulière de Viger durant cette période trouble du Bas-Canada, nous procéderons à une analyse chronologique de son discours public, tout en situant ce même discours par rapport aux événements concrets dans lesquels il s'inscrit. Ainsi, Viger est l'auteur de cinq ouvrages portant sur la politique bas-canadienne et il est actif politiquement de 1808 jusqu'à la veille des rébellions. Ces cinq ouvrages, répartis sur une période allant de 1809 à 1835, constituent les principales sources qui ont servi à la rédaction de cet article. Particulièrement étoffés et tous liés de près à la conjoncture politique dans laquelle ils ont été écrits, ces ouvrages constituent un aperçu fidèle du cheminement politique de Viger, en même temps qu’ils mettent en évidence la continuité de sa pensée durant la période concernée.

Pour commencer, nous brosserons un bref portrait de Viger. Ensuite, nous analyserons ses principaux écrits qui couvrent la période de 1809 à 1822, soit deux pamphlets qui visent à défendre les institutions nationales des Canadiens face aux menaces qui pèsent sur elles. La rhétorique qu'il met en œuvre dans ces textes tient du dialogue et de la persuasion. Cependant, la tentative d'union des deux Canadas de 1822 engendre une rupture importante dans son discours, qui adopte alors une rhétorique plus revendicatrice jusqu’à l'aube des rébellions de 1837. Nous analyserons les trois ouvrages qui suivent ce projet d'union et qui paraissent respectivement en 1831,1834 et 1835 .

\section{Denis-Benjamin Viger}

Denis-Benjamin Viger est né le 19 août 1774, à l'angle des rues Saint-Paul et Saint-Vincent à Montréal ${ }^{15}$. Il est le fils de Denis Viger,

${ }^{14}$ Charles-Philippe Courtois, "Nation et république chez les patriotes ", dans Courtois et Guyot (dir.), La culture des patriotes, p. 86.

15 Pour une biographie plus poussée de Viger, voir Fernand Ouellet et André Lefort, " Denis-Benjamin Viger ", Dictionnaire biographique du Canada, vol. IX : 1861-1870, 
charpentier et député, et de Périne-Charles Cherrier. Du côté des Cherrier, Viger fait partie d'un clan familial qui marquera fortement l'histoire du Bas-Canada et du Canada-Uni. Parmi ses cousins figurent, notamment, l'évêque de Montréal Jean-Jacques Lartigue, le chef patriote Louis-Joseph Papineau, le premier maire de Montréal Jacques Viger, Louis-Michel Viger, l'un des fondateurs de la Banque du peuple en 1835, ainsi que Côme-Séraphin Cherrier, avocat et homme politique.

Viger fait ses études chez les sulpiciens au collège Saint-Raphaël, situé place Jacques-Cartier, avant de se lancer dans la profession d'avocat. Il fait sa cléricature de 1794 à 1799, notamment chez Joseph Bédard, le père de Pierre-Stanislas Bédard. Il est reçu au barreau en mars 1799 et commence à exercer sa profession d'avocat en se constituant une clientèle. Le 21 novembre 1808, il épouse Marie-Amable Foretier, fille de Pierre Foretier, grand propriétaire foncier de qui Viger et son épouse hériteront la seigneurie de l'île Bizard au terme d'une longue bataille juridique qui se termine en 1842. Ensemble, ils n'auront qu'une seule fille, qui mourra à l'âge de huit mois en 1814.

Viger publie ses premiers articles en 1792, à l'âge de dix-huit ans, dans la Gazette de Montréal, alors un journal bilingue ${ }^{16}$. Ses articles sont une réponse aux propos du marchand britannique John Richardson qui, un an après l'adoption de l'Acte constitutionnel, soutenait dans un article que, pour assurer l'unité de l'Empire britannique, une seule langue devait être permise. Le texte de Richardson s'opposait donc à l'usage de la langue française au Bas-Canada et proposait qu'on autorise uniquement la langue anglaise comme langue officielle. Pour défendre l'usage de la langue française, Viger lui

[En ligne], [http://www.biographi.ca/fr/bio/viger_denis_benjamin_9F.html]; voir également Parizeau, La vie studieuse et obstinée de Denis-Benjamin Viger.

16 Joseph Royal, Biographie de l'Hon. D. B. Viger, Montréal, Imprimé par J. A. Plinguet, [s. d.], p. 6, [En ligne], [http://collections.banq.qc.ca/bitstream/52327/2021877/ 1/57277.pdf]; voir également Lefort, Les deux missions de Denis-Benjamin Viger en Angleterre. 
répondit que l'histoire enseignait que l'imposition de la langue latine avait chaque fois entraîné la révolte des peuples conquis par les Romains. Il posait ainsi les premiers jalons d'une pensée qu'il développerait durant les décennies subséquentes.

En 1804, Viger tente une première fois de se faire élire comme député à l'Assemblée législative du Bas-Canada, mais sans succès. Il se présente à nouveau en 1808 et est élu dans Montréal-Ouest. Cette élection marque le début d'une longue carrière politique au cours de laquelle il sera successivement député de Leinster (1810-1816), député du comté de Kent (1816-1830), conseiller législatif (1830-1837), député sous l'Union dans Richelieu et Trois-Rivières, président du Conseil exécutif du Canada-Uni de 1843 à 1846, pour finalement terminer sa carrière au Conseil législatif du Canada-Uni de 1848 à 1858. Toute sa carrière politique est menée de façon laborieuse et consciencieuse, dans le but de servir son pays. Il écrit lui-même, en 1824 :

Il est des hommes qui ne paroissent pas nés pour passer leur vie dans la dissipation ou les plaisirs. Je suis de ce nombre et ma vie est un peu laborieuse. Encore si l'on pouvait obtenir quelques uns $[s i c][\ldots]$ de ces dédomagements [sic] qui parfois rafraichissent l'âme et ravivent dans cette carrière pénible! Mais enfin il faut faire son devoir ${ }^{17}$.

Il fait également partie de plusieurs comités chargés de traiter de différentes questions d'affaires publiques, économiques ou politiques. En 1823, sur cinquante-cinq comités actifs, Viger est le député membre du plus grand nombre d'entre eux, soit trente ${ }^{18}$.

Cette intense activité publique va de pair avec une intense activité intellectuelle. Sa bibliothèque témoigne de sa grande érudition et de son intérêt pour l'étude de la politique et de la chose publique. Vers la fin de sa vie, elle comptait pas moins de 3000 volumes parmi

17 Lettre de Denis-Benjamin Viger à John Neilson, 14 août 1824, citée dans Lefort, Les deux missions de Denis-Benjamin Viger en Angleterre, p. 8.

18 Lefort, Les deux missions de Denis-Benjamin Viger en Angleterre, p. 14. 
lesquels figuraient des ouvrages de Cicéron, Platon, Machiavel, Malthus, Bossuet, Madame de Staël, Adam Smith, Voltaire, Rousseau, Montesquieu, Joseph de Maistre et plusieurs ouvrages portant sur la constitution britannique ${ }^{19}$. Tout au long de sa vie, les questions politiques occupent la majeure partie de ses activités intellectuelles.

Le début de la carrière politique de Viger se fait dans un climat particulièrement tumultueux où s'opposent la majorité parlementaire canadienne à l'Assemblée et la minorité marchande britannique pour la gestion exclusive des politiques du Bas-Canada. La querelle des prisons éclate en 1805 et met au jour les intérêts économiques distincts des deux principaux groupes nationaux. Pour financer la construction de deux nouvelles prisons à Montréal et à Québec, les Canadiens veulent taxer le commerce alors que les marchands britanniques préconisent plutôt de taxer la propriété terrienne. Cette querelle, où la majorité canadienne l'emporte, n'est qu'un des nombreux conflits qui touchent divers sujets de la politique bas-canadienne et qui exacerbent les intérêts divergents des deux principaux groupes nationaux de la colonie. L'impossibilité pour la minorité coloniale britannique d'imposer ses vues commerciales à une Chambre d'assemblée formée d'une majorité canadienne issue du milieu rural amène les dirigeants coloniaux et la minorité marchande à envisager des moyens de court-circuiter la députation canadienne gênante. L'oligarchie coloniale regrette d'avoir adopté l'Acte de 1791, qui a séparé le Bas et le Haut-Canada et qui a octroyé le régime représentatif aux Canadiens par la création de la Chambre d'assemblée. Elle entend trouver des moyens de remédier à ce problème.

Plusieurs solutions sont envisagées durant le premier quart du XIX ${ }^{\mathrm{e}}$ siècle, notamment entre 1808 et 1810 et en 1822 . On songe à abolir les institutions canadiennes comme les lois civiles françaises, le régime seigneurial ou l'usage de la langue française dans l'enseignement. On envisage aussi d'augmenter l'immigration britannique

19 Parizeau, La vie studieuse et obstinée de Denis-Benjamin Viger, p. 155 ; voir également Lefort, Les deux missions de Denis-Benjamin Viger en Angleterre, p. 7. 
pour noyer progressivement la population canadienne et unir les deux Canadas ${ }^{20}$. Ces projets et ces menaces envers les Canadiens sont repris dans les journaux de la colonie, notamment dans Le Canadien et le Quebec Mercury, qui se querellent pour défendre les positions de leur groupe respectif. Dans la foulée de ces divers projets menaçant directement le groupe national canadien et ses institutions, Viger écrit et prend la parole dans le but de contrer les projets d'union et d'assimilation de la population canadienne. Comme il l'affirme lui-même : "J'aime mon pays et tous ceux qui le servent ${ }^{21}$. " C'est donc dans cette optique civique qu'il faut comprendre l'engagement de Viger.

\section{Le temps de la persuasion (1809-1822)}

Écrit en 1809 et longuement intitulé Considerations sur les effets qu'ont produit en Canada, la conservation des etablissemens du pays, les moeurs, l'education, etc. de ses habitans; et les consequences qu'entraineroient leur decadence par rapport aux interets de la Grande Bretagne, l'ouvrage de Viger, d'une cinquantaine de pages, tente, comme son titre l'indique, d'expliquer aux Britanniques pourquoi il est dans leur intérêt de conserver les institutions canadiennes. Viger répond particulièrement à un texte paru dans le Quebec Mercury sous la plume de Scévola, qui préconisait d'abolir l'enseignement en français dans les collèges canadiens. De manière générale, son texte se veut également une réponse aux articles des éditeurs du même journal, qui réclament fréquemment l'abolition des institutions des Canadiens, comme les lois civiles, le régime seigneurial, la religion catholique ou la langue française.

${ }^{20}$ Pour un résumé de ces projets d'assimilation, voir Maurice Séguin, Histoire de deux nationalismes au Canada, Montréal, Guérin, 1997, p. 143-164.

21 Denis-Benjamin Viger, Considerations sur les effets quiont produit en Canada, la conservation des etablissemens du pays, les mours, l'education, etc. de ses habitans; et les consequences qu'entraineroient leur decadence par rapport aux interets de la Grande Bretagne, Montréal, Imprimé chez James Brown, Libraire, 1809, p. ii, [En ligne], [http://collections.banq.qc.ca/bitstream/52327/2036283/1/141790.pdf]. 
Tout en insistant sur la fidélité passée de la population canadienne à la Grande-Bretagne lors de l'Indépendance américaine, fidélité justifiant la reconnaissance de ses droits et de ses institutions, Viger s'emploie à montrer les avantages futurs découlant de leur maintien. Il défend également les Canadiens en rappelant qu'ils sont des sujets anglais jouissant, au même titre que n'importe quel Britannique, des libertés anglaises. Viger use donc d'un discours britanniste mis en lumière par plusieurs historiens des rébellions, allant de Lionel Groulx à Louis-Georges Harvey ${ }^{22}$. Ce dernier a d'ailleurs souligné le fait que la rhétorique du discours patriote s'abreuvait à l'humanisme civique inspiré de l'héritage gréco-romain et de l'exemple de la République romaine $^{23}$. La référence aux Anciens dans les discours politiques servait de caution aux actions du présent. À cet égard, Viger cite de nombreux exemples tirés de l'histoire, comme Carthage, Rome, Guillaume le Conquérant, etc., qui lui permettent d'insister sur l'importance de respecter les populations vaincues en montrant ce qu'il est advenu de ceux qui ont omis de le faire. Pour cet avocat de formation, l'histoire agit ici à titre de prescription, de jurisprudence pour aider à juger des actions à entreprendre dans le présent. Il plaide que les empires qui ne respectent pas les mœurs des populations conquises finissent par être confrontés à des révoltes, des tumultes et des problèmes qui entraînent souvent leur décadence.

Notons toutefois que si le discours de Viger est imprégné d'influences classiques et que ce dernier use abondamment d'axiomes moraux tout au long de sa carrière, cet humanisme civique est mis au service d'un discours conservateur et constitutionnel plutôt que républicain. De même, il s'accompagne de l'influence de penseurs conservateurs et contre-révolutionnaires, comme Edmund Burke et Joseph de Maistre.

22 Lionel Groulx, "Dossier : le "britannisme" des "patriotes" ", Revue d'histoire de l'Amérique française, vol. 5, no 3 (décembre 1951), p. 416-425; Harvey, Le printemps de l'Amérique française.

${ }^{23}$ Harvey, Le printemps de l'Amérique française, chapitres I et II. 
En effet, pour justifier la sauvegarde des institutions canadiennes, Viger s'applique à jouer sur le même terrain que les Britanniques en utilisant les thèses d'un de leurs plus illustres penseurs : Edmund Burke. Dans ses Réflexions sur la révolution de France ${ }^{24}$, celui qui est considéré comme le père du conservatisme moderne pourfend la Révolution française, qu'il considère comme une hérésie visant à faire tabula rasa du passé et à ériger un homme nouveau au sein d'un monde nouveau. Pour Burke, la constitution d'un pays doit s'arrimer à l'histoire et aux traditions du pays dans lequel elle s'inscrit. En ce sens, il s'oppose à l'universalisme abstrait des Lumières incarné dans les idéaux de la Révolution française et la Déclaration des Droits de l'Homme et du Citoyen. L'homme ne peut faire table rase du passé, imposer sa propre volonté et établir un nouveau contrat social par une nouvelle constitution sans entraîner de graves conséquences, comme l'ont fait les Français, qui ont engendré la Terreur. En outre, face à l'égalitarisme abstrait promu par les révolutionnaires français, Burke défend une conception hiérarchique de la société conforme au gouvernement mixte en usage en Angleterre :

Dans toutes les sociétés composées de différentes classes de citoyens, il faut qu'il y en ait une qui tienne le premier rang. C'est pourquoi les niveleurs ne font que pervertir l'ordre naturel des choses; ils surchargent l'édifice social en plaçant tout en haut ce qu'il faut mettre en bas, si l'on veut que le bâtiment soit solide ${ }^{25}$.

Chez Burke, la constitution d'un pays est le fruit des générations qui se sont succédé au cours des époques. Les révolutionnaires français " opposent un progrès incertain aux certitudes du passé. Ils préconisent des innovations, dont on ne connait pas les lendemains, à la sagesse accumulée par l'expérience ${ }^{26} »$.

${ }^{24}$ Edmund Burke, Réflexions sur la révolution de France, préface de Philippe Raynaud, Paris, Hachette, 1989.

25 Ibid., p. 62.

26 Jean-Marc Piotte, "Edmund Burke (1729-1799) ", dans Les grands penseurs du monde occidental : l'éthique et la politique de Platon à nos jours, Montréal, Éditions Fides, 2005, p. 310. 
Ainsi, Viger utilise les thèses de Burke, qui défendait la société anglaise contre les idéaux de la Révolution française, en les appliquant à la société canadienne contre l'impérialisme de l'oligarchie britannique. Selon Viger, l'Angleterre a fait preuve de sagesse en 1791 en concédant un territoire séparé au peuple canadien et en maintenant les institutions, la langue et les mœurs issues de la Nouvelle-France. Mais elle ferait erreur en revenant sur sa décision et en enlevant ces droits, en unissant les deux Canadas ou en tentant d'assimiler les Canadiens. Il critique notamment le désir de certains de faire immigrer des réfugiés américains dans les townships et qui, pour ce faire, préconisent d'abolir les lois civiles françaises et d'établir le système de franc et commun soccage plutôt que le régime seigneurial. « Il faudroit, suivant ces penseurs, substituer à la population de ce pays une population nouvelle, digne de l'habiter, et d'en tirer parti ${ }^{27}$. » Il invite à ce "qu'on lise les sages réflexions [de] l'illustre Burke ${ }^{28}$ ". Pour lui, à l'instar de Burke : «C'est sur l'expérience qu'il faut établir les maximes de la politique et du gouvernement; c'est sur cette base qu'il faut juger toutes les questions qui intéressent l'humanité et les nation $s^{29}$." Or, à cet égard, les Canadiens ont " des règles sures, fondées sur l'expérience de notre propre pays ${ }^{30} \%$. Le Bas-Canada de Viger constitue le territoire de la population canadienne d'origine française. Celle-ci possède ses propres institutions et elles doivent donc être implantées sur l'ensemble du territoire, y compris dans les townships, où la population canadienne en croissance devra tôt ou tard s'installer.

Dans l'argumentation conservatrice burkienne dont se sert Viger, tenter d'assimiler la population canadienne serait une erreur aux conséquences incalculables : ce serait « une folie ridicule de frapper de stérilité un fond riche pour courir après des espérances incertaines,

\footnotetext{
27 Viger, Considerations, 1809, p. 30.

28 Ibid., p. 9.

${ }^{29}$ Ibid., p. 7.

30 Ibid., p. 5.
} 
fondées sur un avenir trompeur ${ }^{31}$ ». La doctrine conservatrice, voulant que l'homme doive se conformer à ce qui est, sert donc l'argumentation de Viger dans la défense du donné qu'est la population canadienne au Bas-Canada. Le conservateur " revendique le concret, le réel, le continu, contre la fiction abstraite des constructions ex nibilo ${ }^{32}$ ”.

Ainsi, Viger s'attarde à montrer aux Britanniques le bien-fondé de conserver une population canadienne forte et homogène avec ses institutions face aux Américains : « [...] je ferai voir même de manière à ne laisser aucun doute, que la conservation de cette langue en ce pays, est même, politiquement parlant, avantageuse aux intérêts Britanniques ${ }^{33}$. "Bien conscient des enjeux géostratégiques de l'Angleterre liés à la possession de ses colonies d'Amérique du Nord pour contrer la montée en puissance des jeunes États-Unis, Viger soutient qu'un bloc canadien d'origine française au Bas-Canada constitue une meilleure assurance pour l'Angleterre de conserver ses colonies que si elle s'emploie à l'assimiler ou à le noyer dans une masse de colons britanniques. Des colons britanniques seraient plus susceptibles de se fondre dans la jeune république, car plus près des Américains culturellement. Ainsi, pour Viger, l'intérêt des Canadiens, soit de conserver leurs institutions, leur langue et leur culture, rencontre l'intérêt britannique, face à la menace représentée par les États-Unis.

Ce texte de 1809 connait un certain succès auprès du public lettré et des hommes politiques du Bas-Canada ${ }^{34}$. Toutefois, le projet d'union de 1810 n'aboutit pas, et la guerre de 1812 contre les ÉtatsUnis est l'occasion de calmer les tensions entre les deux groupes nationaux du Bas-Canada. Viger participe à la guerre à titre de capitaine de milice, et se plaint auprès des Anglais du fait que les

${ }^{31}$ Ibid., p. 35.

32 Michel Winock, "L'héritage contre-révolutionnaire ", dans Histoire de l'extrêmedroite en France, Paris, Éditions du Seuil, 1994, p. 30.

${ }^{33}$ Viger, Considerations, 1809, p. 28.

${ }^{34}$ Voir Lefort, Les deux missions de Denis-Benjamin Viger en Angleterre, p. 18. 
ordres doivent être donnés en anglais aux miliciens ${ }^{35}$. Le reste de la décennie 1810 voit progressivement le leadership du Parti canadien passer des mains du clan de Québec à celles du clan montréalais, mené par Louis-Joseph Papineau et Viger. Le Canadien du 14 février 1818 soutient même que Viger et Papineau sont parmi les gens qui ont le plus d'influence sur l'opinion publique bas-canadienne ${ }^{36}$. Selon Fernand Ouellet, les deux cousins sont les principaux meneurs du Parti canadien au tournant des années $1820^{37}$.

C'est dans ce contexte où son influence prend de l'ampleur que Viger effectue un voyage aux États-Unis en 1819, voyage qui lui inspire la tenue d'un journal dans lequel il transcrit, sous forme de lettres, les entretiens qu'il a eus avec des Américains. Conscient que certains marchands britanniques et membres de l'exécutif colonial cherchent toujours à unir les Canadas ou à abolir les institutions canadiennes, il fait paraître certaines de ces lettres dans les journaux du Bas-Canada, mais publie la plus longue dans un ouvrage de 46 pages, en $1826^{38}$. Cet ouvrage, qui reprend essentiellement l'argumentation développée dans son volume de 1809, est le résumé d'une conversation qu'a eue Viger dans laquelle son interlocuteur soutenait que les Canadiens ne seraient jamais de bons sujets britanniques tant "que le Bas-Canada continuera d'être François par sa langue, sa religion, ses usages et ses mœurs ${ }^{39}$ ". Pour le bien de l'Empire britannique, cet interlocuteur préconisait donc l'assimilation de la population canadienne. À l'aide d'exemples tirés de l'histoire, telles que les îles de Jersey, dont les habitants ont conservé leurs particularités culturelles et font paisiblement partie de la Grande-Bretagne, Viger montre que les peuples restent fidèles à leurs souverains ou leurs conquérants lorsque « ces peuples n'ont rien perdu de ce qui attache

\footnotetext{
35 Ibid., p. 10.

${ }^{36}$ Le Canadien, 14 février 1818, dans Lefort, Les deux missions de Denis-Benjamin Viger en Angleterre, p. 12.

37 Ouellet et Lefort, "Denis-Benjamin Viger ".

38 Viger, Analyse d'un entretien.

39 Ibid., p. 9.
} 
les hommes à la société dont ils sont membres ${ }^{40} »$. En contrepartie, Viger cite l'exemple de l'Irlande, dont l'histoire "présente une suite non interrompue d'actes de tyrannie, d'un côté, de révoltes, de l'autre : de brigandages, de spoliations, de meurtres ». La raison de ces désordres irlandais est claire pour Viger : "On n’a jamais mis plus de constance dans l'exécution d'un projet que dans celui d'anglifier l'Irlande ${ }^{41}$. " La conclusion que tire Viger des divers exemples qu'il expose est qu'il est justifié de conserver les particularités nationales et culturelles des Canadiens, autant pour les Canadiens eux-mêmes que pour la paix et la stabilité dans la colonie britannique : "Les peuples restent toujours attachés à l'ordre de choses qui assure la jouissance de leurs droits ${ }^{42}$." Si les droits et les institutions des Canadiens sont respectés, l'Angleterre n'a pas à craindre de voir le peuple canadien se soulever contre elle, soutient Viger.

\section{La nation canadienne chez Viger}

Ce qui doit surtout retenir notre attention dans les écrits de Viger de 1809 et 1819 est la conception de la nation qu'il y développe. Car, au-delà de l'argumentation de surface utilisée pour défendre les institutions canadiennes, ses écrits permettent de mettre en lumière l'idée qu'il se fait des nations, en général, et de la nation canadienne, en particulier. Fernand Ouellet soutient que le nationalisme des patriotes était avant tout motivé par la défense d'intérêts de classe chez les petites élites libérales, qui auraient cherché à conserver leur ascendant sur la société canadienne d'Ancien Régime ${ }^{43}$. Ouellet, qui a intériorisé le point de vue britannique sur la question, comprend mal le nationalisme présent au sein du mouvement patriote et l'attachement de certains de ses protagonistes à leurs institutions et

40 Ibid., p. 17.

41 Ibid., p. 17-18.

42 Ibid., p. 20.

43 Fernand Ouellet, Histoire économique et sociale du Québec, t. 2, Montréal, Éditions Fides, 1971, p. 414. 
à leur culture particulière. C'est pourquoi il considère ce nationalisme comme une force rétrograde allant à l'encontre du progrès. La notion de progrès est implicitement comprise chez Ouellet dans le sens de la pensée des Lumières écossaises défendues par l'oligarchie marchande, selon laquelle une société moderne est fondée sur l'expansion infinie du commerce et la domination de l'homme sur la nature ${ }^{44}$. C'est sous un autre angle que nous allons aborder la question. Si, à première vue, la défense des institutions canadiennes, particulièrement le régime seigneurial, semble effectivement servir les intérêts de Denis-Benjamin Viger, cette défense se fait également en vertu d'une véritable conception holistique et culturelle de la nation canadienne, qu'il se représente liée au territoire du BasCanada. Cette nation issue de la colonisation française possède ses propres institutions et particularités héritées de son passé, comme le droit civil, le système seigneurial, la religion catholique, la langue française et les us et coutumes qui en sont la base. Chacune de ces institutions répond à une certaine logique au sein de la nation, et toutes sont complémentaires. Elles s'insèrent dans l'ordre national, que Viger conçoit comme un «systême [sic] politique ». Ainsi, à ceux qui veulent départir les Canadiens de certaines de leurs particularités nationales pour les assimiler, pour favoriser l'immigration d'origine britannique, pour s'approprier plus facilement le sol ou, encore, pour promouvoir des projets économiques de grande envergure commerciale comme la voie maritime du Saint-Laurent, Viger répond :

Tout se tient dans le systême politique : ôtez une pièce qui souvent ne paroît aux yeux de l'observateur superficiel qu'un hors d'œuvre ou un ornement superflu, vous en dérangez souvent, par cela même toute l'économie. C'est la clef d'une voute; la petitesse de ses dimensions vous la fait mépriser, vous l'enlevez, l'édifice s'écroule, et écrase sous ses ruines l'imprudent architecte ${ }^{45}$.

${ }^{44}$ Fernand Ouellet, "Nationalisme canadien-français et laïcisme au XIX ${ }^{\mathrm{e}}$ siècle ", dans Jean-Paul Bernard (dir.), Les idéologies québécoises au $19^{\circ}$ siècle, Montréal, Boréal express, 1973, p. 48 ; pour la pensée écossaise, voir la préface de Philippe Raynaud, dans Burke, Réflexions sur la révolution de France, p. Lxx.

45 Viger, Considerations, 1809, p. 35. En italique dans le texte original. 
Ce passage indique bien que, pour Viger, une nation forme un ensemble culturel cohérent forgé par l'histoire. C'est notamment le cas de la nation canadienne. Une nation constitue un système ordonné dont les institutions sont le produit du temps et possèdent leur propre logique interne, une sorte d'équilibre qui est propre à chaque nation. En enlevant ne serait-ce qu'une partie infime des composantes de l'ordre national, l'équilibre de la nation est rompu et «il faut le sacrifice de plusieurs générations à la suite les unes des autres pour rétablir l'équilibre une fois détruit ${ }^{46}$ ».

Au début du XIx ${ }^{e}$ siècle, face à la nation contractualiste à la française, qui constitue une collection d'individus porteurs de droits et un cadre pour l'émancipation de ceux-ci, la nation holistique à l'allemande s'affirme et mise plutôt sur la communauté culturelle forgée par l'histoire ${ }^{47}$. D'un côté, un nationalisme civique, rationnel et universaliste et, de l'autre, un nationalisme culturel, traditionnaliste et organique. Le premier est associé à un phénomène politique présent dans des pays pourvus d'État tels que la France, les États-Unis et la Suisse, alors que le second concerne des pays dépourvus d'État tels que la Pologne, l'Italie et l'Allemagne ${ }^{48}$. Souvent soumises à la domination de puissances impériales étrangères, les nations dépourvues d'État réagissent aux agressions et aux menaces dont elles sont victimes en développant un mécanisme de défense misant sur leurs particularités culturelles collectives. Comme le souligne le sociologue Alain Dieckhoff, le holisme de la nation culturelle n'implique "pas la négation de l'individualisme, mais le transfert de celui-ci au niveau collectif $^{49}$ ". Selon la conception culturelle et historique, la nation est un tout, un individu collectif. C'est dans cette logique qu'il faut comprendre le nationalisme de Viger et la systématisation qu'il fait

\footnotetext{
${ }^{46}$ Ibid.

47 Voir Alain Dieckhoff, "Nationalisme politique contre nationalisme culturel? ", dans Alain Dieckhoff et Christophe Jaffrelot (dir.), Repenser le nationalisme, Paris, Presses de sciences po, 2006, p. 106.

48 Hermet, "Nationalisme ", p. 802.

49 Dieckhoff, "Nationalisme politique contre nationalisme culturel? ", p. 108.
} 
de la nation canadienne devant les menaces qui pèsent sur ce groupe national auquel il s'identifie. La conception qu'il se fait de la nation canadienne est holistique, en ce sens qu' elle forme un tout organique qu'il apparente à un "systême ». Elle est également culturelle, puisque ce tout organique se définit par ses caractéristiques culturelles : la langue, la religion, les institutions et les us et coutumes qui en sont les fondements.

Bien qu'à l'époque de Viger le nationalisme holistique et culturel ait été théorisé par des penseurs allemands comme Johann Gottfried Herder et Johann Gottlieb Fichte, des penseurs français comme Louis de Bonald et Joseph de Maistre ont également défendu cette conception de la nation dans leur critique de la Révolution française et des principes philosophiques qui en sont à la base. Lecteur de Joseph de Maistre - pour qui « les nations ont une âme générale et une véritable unité morale qui les constituent ce qu'elles sont ${ }^{50}$ ", Viger adhère à la conception holistique de la nation chez de Maistre, sans pour autant adhérer aux idées théocratiques du penseur français. Le conservatisme de Viger est modéré et s'apparente davantage au whiggisme de Burke qu'à la pensée d'Ancien Régime de Joseph de Maistre en ce que, tout comme Burke et comme nous le verrons dans les lignes qui suivent, Viger croit aux vertus d'une constitution mixte pour contrebalancer le pouvoir royal ${ }^{51}$. De plus, si Viger considère la religion catholique comme une des données fondamentales de la culture canadienne et qu'il défend les possessions de l'Église au BasCanada en la défendant en tant qu'ordre ${ }^{52}$, il est loin d'adhérer au théocratisme de Joseph de Maistre, et sa pensée catholique s'apparente davantage au libéralisme chrétien d'un Félicité de Lamennais ${ }^{53}$. En

${ }^{50}$ Cité dans Frédéric Boily, La pensée nationaliste de Lionel Groulx, Sillery, Éditions du Septentrion, 2003, p. 24.

51 Yves Chiron, Edmund Burke et la Révolution française, Paris, Téqui, 1987, p. 11.

52 Lors de ses missions en Angleterre, Viger se fait le porte-parole de l'Église catholique bas-canadienne et défend ses intérêts. Voir « Notes secrètes à D. B. Viger, pour son séjour à Londres ", Correspondance entre D.-B. Viger et $\mathrm{M}^{\mathrm{gr}}$ Jean-Jacques Lartigue, Archives de l'archevêché de Montréal, 901.023, 828-1.

53 Voir ce qu'en dit Lamonde, Histoire sociale des idées au Québec, p. 294. 
effet, Viger n'hésite pas à remettre en cause l'autorité papale pour défendre Lamennais lors de l'affaire de la Pologne de même que celle de son cousin Jean-Jacques Lartigue à la veille des troubles de $1837^{54}$. L'influence exercée par de Maistre sur Viger se limite donc essentiellement à sa pensée nationaliste, selon laquelle la nation est un tout porteur d'un certain "génie ".

Selon cette conception, une nation et une société sont ordonnées et se régulent de la même façon que le mouvement des astres, que les saisons se succèdent ou que se régule le corps humain, dans un ordre parfait ${ }^{55}$. Tout comme l'univers (le macrocosme) possède ses propres lois, les sociétés humaines (des microcosmes) ont les leurs, un ordre national, auquel l'homme doit se conformer. Pour comprendre les lois qui régissent l'agir humain et les sociétés, l'histoire joue ici le rôle de révélatrice. C'est donc à ce titre que les exemples que Viger tire de l'histoire agissent comme des prescriptions, comme des guides dans la marche à suivre dans le présent.

Selon la vision de Viger, l'abolition des lois civiles françaises ou du régime seigneurial, comme le proposent les membres de l'oligarchie coloniale, engendrera le dérèglement des autres institutions et du système national en entier, ainsi que l'a montré l'histoire : "L'expérience de tous les siècles prouve que ces altérations, ces changemens imprudens, entraînent presque toujours la dissolution des corps politiques $[\ldots]^{56}$. " Chez lui, les institutions nationales d'un peuple ne sont pas des éléments interchangeables que l'on peut enlever sans engendrer ensuite des conséquences sur l'ensemble de la société. Elles sont complémentaires et interdépendantes les unes les autres, elles forment "les liens de l'édifice d'une société. Otez à un peuple ces élémens d'union, et il perd ses forces, il languit, il meurt ${ }^{57}$ ». Ainsi,

${ }^{44}$ Voir Fernand Ouellet, "Le mandement de $\mathrm{M}^{\mathrm{gr}}$ Lartigue de 1837 et la réaction libérale ", Bulletin des recherches historiques, vol. 58, n² 2 (avril-juin 1952), p. 97104.

55 Frédéric Boily, Le conservatisme au Québec, Québec, Les Presses de l'Université Laval, 2010, p. 20.

56 Viger, Considerations, 1809, p. 35.

57 Viger, Analyse d'un entretien, p. 32. 
cette conception holistique attribue une fonction particulière à chacune des institutions au sein du système national en entier, lui assurant son bon fonctionnement.

La conception de la nation chez Viger se traduit également dans son admiration de la constitution mixte britannique, qui permet aux différentes composantes d'une société de se compléter et de s'équilibrer dans un ordre parfait :

La Constitution britannique est peut-être la seule où les droits et les intérêts des différentes branches dont la société est composée sont tellement ménagés, si sagement opposés et tous ensemble liés les uns aux autres, qu'elles s'éclairent mutuellement et se soutiennent par la lutte même qui résulte de l'exercice simultané des pouvoirs qui sont confiés ${ }^{8}$.

Le parfait équilibre et l'harmonie sont ce que Viger recherche dans une société et il constate la présence de ces caractéristiques dans la constitution mixte britannique. L'équilibre entre la monarchie (exécutif), l'aristocratie (Conseil législatif) et le peuple (Assemblée) convient à la conception ordonnée et systémique que se fait Viger d'une société. Louis-Georges Harvey a d'ailleurs fait remarquer que la variante britannique de l'humanisme civique conforta le constitutionnalisme dans l'équilibre apporté par les trois branches du Parlement ${ }^{59}$. En se contrebalançant les unes les autres, les trois branches du gouvernement mixte évitaient mutuellement de se corrompre et de corrompre ensuite la société, à l'instar de ce que prévoyait la typologie aristotélicienne des régimes politiques. Les archives personnelles de Viger sont également parsemées de réflexions théoriques qui comparent les monarchies absolues aux monarchies constitutionnelles et qui louangent ces dernières et leur sain équilibre, dont les aspects constitutionnels empêchent le monarque de prendre

${ }^{58}$ Le Canadien, 4 novembre 1809, cité dans Fernand Ouellet, Le Bas-Canada 1791-1840 : changements structuraux et crise, Ottawa, Éditions de l'Université d'Ottawa, 1980, p. 156.

59 Harvey, Le printemps de l'Amérique française, p. 33. 
des décisions arbitraires et agissent "pour les peuples comme garanties (et pour) les gouvernemens comme moyens de durée ${ }^{60} "$.

De plus, en permettant aux Canadiens d'être représentés à l'Assemblée, la Constitution de 1791 leur permettait de se gouverner selon leur propre génie et "de conserver [leurs] loix, [leurs] usages, [leur] langue, [leur] culte ${ }^{61}{ }^{\prime}$. C'est pourquoi « [l]'établissement d'une constitution libre [en 1791] en ce pays a fait naître des sentimens de reconnoissance plus vifs encore ${ }^{62} \%$. Cependant, comme nous le verrons dans les lignes qui suivent, le problème que décèle progressivement Viger et qui se traduit dans ses revendications à l'aube des rébellions de 1837 réside dans le fait que le Conseil législatif du Bas-Canada est largement aux mains d'une oligarchie britannique. Guidée par ses passions et ses antipathies nationales, celle-ci trouble l'ordre de la nation canadienne, empêche les Canadiens de véritablement se gouverner; de là vient, pour Viger, la source du problème du Bas-Canada et la raison pour laquelle la constitution britannique n'est pas bien appliquée.

La conception de la nation et de l'ordre social chez Viger s'inscrit donc dans le courant romantique et conservateur qui émerge après les soubresauts de la Révolution française en Europe. Ce courant adhérait à une tout autre conception de l'homme et de la vie que ce que proposait le progressisme libéral des Lumières incarné dans les idéaux de la Révolution française et la Déclaration des Droits de l'Homme et du Citoyen. Pour le conservateur, l'homme des Lumières, guidé par l'hubris, enfreint les lois naturelles et joue à Dieu avec les sociétés humaines et les constitutions politiques, comme l'ont fait les Français. A contrario, la conception conservatrice de l'homme se veut plus humble face à la vie. Comme Burke le soutenait, l'homme doit se conformer à ce qui est, à l'ordre national ou sociétal dans lequel il vit, à ses lois et il ne peut en faire fi sans encourir de graves

${ }^{60}$ Réflexions politiques, Bibliothèque et Archives Canada (ci-après BAC), Fonds Denis-Benjamin Viger, MG24-B6, vol. 8, \#4367-4375.

${ }^{61}$ Viger, Analyse d'un entretien, p. 31.

${ }^{62}$ Viger, Considerations, 1809, p. 40. 
conséquences. Les changements dans une société doivent se faire d'eux-mêmes, graduellement et sans rupture trop brutale, car une rupture brusque engendre des désordres et des tumultes : « [...] il n'y avoit que les changemens qui s'opéroient graduellement, comme d'eux-mêmes et sans efforts, qui n'enfantoient pas ces sujets de collision, et trop souvent les convulsions qui faisoient le malheur des peuples $[\ldots]^{63}$. " Ainsi, Viger adhère à la critique des idées des Lumières, de la Révolution française et à la conception conservatrice de l'homme, telles qu'avancées par Burke et de Maistre : "Ces idées rebattues de perfectibilité de l'espèce humaine et des gouvernemens mises au jour par les écrivains François [...] ont été éprouvées au creuset de l'expérience ${ }^{64}$. » Ici, il importe de préciser qu'il ne faut pas voir en Viger un pessimiste à l'égard de la nature humaine. Il croit à une forme de progrès chez l'humain. Seulement, cette évolution doit se faire progressivement, sans être provoquée par des révolutionnaires trop pressés. C'est en ce sens qu'il plaide fréquemment au cours de sa carrière en faveur de l'éducation du peuple et l'amélioration des techniques, principalement en agriculture.

En vertu de sa conception holistique et systémique, Viger considère les choses dans leur globalité plutôt que dans leur singularité, et l'être humain comme une partie intégrante de l'univers, du monde et de la société qui l'entoure. En ce sens, cette vision s'oppose à l'humanisme libéral des Lumières qui, sous prétexte d'universalisme, extirpe et dissocie l'homme du monde dans lequel il vit pour le considérer comme unique valeur. La nation, selon Viger, n'est pas une collection d'individus liés par un contrat social, elle est un tout culturel ordonné par ses institutions, ses us et coutumes et forgé par l'histoire. Ainsi, en utilisant l'argumentation de Burke, Viger soutient que les Britanniques, plutôt que de chercher à transformer ce tout culturel canadien, doivent le respecter et s'y conformer.

${ }^{63}$ Viger, Analyse d'un entretien, p. 42.

${ }^{64}$ Viger, Considerations, 1809, p. 10. 
L'opposition de Viger à une conception trop libérale et individualiste de la société est bien exprimée dans cette citation tirée d'un ouvrage de 1831 : « Les lois, les institutions civiles ou religieuses d'un peuple, ses coutumes, ses usages ne sont-ils pas ce qu'il y a de plus important dans ce qui lui appartient, et ses droits à ce genre de propriété ne sont-ils pas plus sacrés en raison de cette importance là même ${ }^{65}$ ? " C'est le mot " propriété » ici qui doit retenir l'attention. Pour Viger, la société étant un tout, les institutions nationales forment la propriété collective du peuple canadien, et " [t]outes les nations ont une prédilection fort naturelle pour leurs établissemen $s^{66}{ }$. C'est pourquoi en mettant l'accent sur l'ensemble de la société plutôt que sur l'individu, le nationalisme holistique et culturel de Viger ne peut concevoir qu'on détruise les institutions nationales canadiennes issues de l'histoire de la nation, comme le régime seigneurial, au nom d'une conception libérale et individualiste de la propriété. En 1826, il s'inquiétait d'ailleurs des « habitudes du barreau qui retrécissent quelquesfois les idées en les concentrant sur des questions de droits individuels ${ }^{67}$ \%. Il préfere l'établissement de lois et de normes générales qui permettent d'assurer l'ordre du système national en régissant certains comportements humains et en balisant les vices de la nature humaine.

Soulignons toutefois que le nationalisme conservateur de Viger n'est pas incompatible avec une certaine forme de libéralisme. Seulement, ce libéralisme s'applique à la nation canadienne avant de s'appliquer aux individus. Fernand Ouellet a d'ailleurs fait remarquer que le principe des nationalités représentait, "pour une part, la transposition au niveau collectif de certains aspects de la philosophie

65 Denis-Benjamin Viger, Considérations relatives à la dernière révolution de la Belgique, Montréal, [s. n.], 1831, p. 10.

${ }^{66}$ Viger, Analyse d'un entretien, p. 44.

${ }^{67}$ Lettre de Denis-Benjamin Viger à John Neilson, 2 novembre 1826, BAC, Collection John Neilson et famille, MG24-B1, vol. 33, Correspondance de Denis-Benjamin Viger, Bobine C15776, [En ligne], [http://heritage.canadiana.ca/ view/oocihm.lac_reel_c15776/649?r=0\&ss=3], images 648-649. 
des droits individuels ${ }^{68}$ ". C'est dans cette logique que Viger transpose le droit de propriété, attribué à l'individu dans la société libérale, au peuple canadien dans sa conception holistique. Ainsi, les institutions nationales, les mœurs et la langue constituent des propriétés inaliénables de la nation canadienne. Celle-ci n'est pas conçue comme une masse d'individus citoyens possédant des droits individuels, bien qu'ils en possèdent, mais elle est avant tout perçue comme un tout complémentaire, comme un individu collectif, lui-même tributaire de droits naturels, sacrés et inaliénables.

La conséquence de la conception holistique et systémique de la nation chez Viger est manifeste : les Canadiens doivent constituer une entité politique séparée du peuple anglais. C'est pourquoi Viger considère que l'Angleterre a été sage de séparer les deux Canadas en 1791 et de laisser les Canadiens gérer leurs propres institutions en autorisant l'élection de leurs représentants à l'Assemblée. C'est en ce sens qu'il s'oppose à l'union des deux Canadas de même qu'à ce que les institutions britanniques remplacent les institutions canadiennes dans les townships. Selon sa vision des choses et comme l'exemple de la Belgique lui permettra de le constater en 1831, deux systèmes nationaux parallèles ne peuvent coexister au sein du Bas-Canada ou sur un même territoire politique sans que l'un n'entrave l'autre, d'autant plus que Viger est conscient des desseins de l'oligarchie coloniale qui encourage l'immigration de masse britannique dans les townships. Unir deux ordres nationaux ne peut qu'entraîner le désordre et des troubles. Il s'en explique ainsi en 1819 :

Je demanderai d'abord combien de temps exigera la fusion de ces matières hétérogènes? [...] N'y aura-t-il pas entre les anciens et les nouveaux venus quelqu'opposition de vues, de sentimens, d'intérêts? [...] La conséquence la moins malheureuse qui suivroit inévitablement l'exécution de ce projet, ce seroit d'abord, sinon la discorde pour prélude à une confusion universelle, assurément

${ }^{68}$ Ouellet, « Nationalisme canadien-français et laïcisme au XIX ${ }^{\mathrm{e}}$ siècle », p. 46. 
pendant longtemps l'engourdissement de l'apathie, une indifférence profonde pour ceux qui auroient fait éclore ce cahos $[s i c]^{69}$.

Si, comme le soutient Yvan Lamonde, Louis-Joseph Papineau était le "pourfendeur des "distinctions nationales"70 ", ce n'est pas le cas de Denis-Benjamin Viger. Ce dernier reconnaît des distinctions culturelles et institutionnelles à chaque nation, au même titre que les individus diffèrent les uns des autres en fonction de leur vécu, de leurs expériences, de leur cheminement, de leurs intérêts, etc.

Ainsi, de 1809 à 1819 et même par la suite, les attaques de l'oligarchie coloniale et les menaces qui pèsent sur les institutions canadiennes amènent Viger à tenir un discours fondamentalement nationaliste. Ses écrits tournent autour de la question nationale et s'attardent à défendre la conservation de la nation et de ses institutions. D'ailleurs, des causes affectives le poussent également à vouloir sauvegarder les institutions et la nation canadiennes : "Lamour de la patrie, l'affection pour tous les objets qui s'y rattachent ne doivent pas être placés au rang des illusions ${ }^{71}$. "Viger conçoit les institutions canadiennes comme un héritage issu des ancêtres de la NouvelleFrance. Pour les Canadiens, elles forment « la partie la plus précieuse, la portion la plus chère de l'héritage qu'ils tiennent de leurs pères ${ }^{72}$ ".

Cependant, devant l'échec de la persuasion et l'évidence que le projet de britanniser le Bas-Canada est progressivement mis en application avec l'immigration de masse, la non-application des lois civiles dans les townships et, surtout, avec le projet d'union de 1822, le discours de Viger change considérablement pour adopter un ton plus résolu. Le ton est alors à l'analyse sérieuse du problème et à la recherche de remèdes. La révolution belge constituera ainsi un cas des plus intéressants pour Viger.

\footnotetext{
69 Viger, Analyse d'un entretien, p. 36-37.

${ }^{70}$ Lamonde, Histoire sociale des idées au Québec, p. 219.

${ }^{71}$ Viger, Analyse d'un entretien, p. 32.

72 Ibid., p. 35.
} 


\section{Analyse du problème des Canadiens (1822-1834)}

L'année 1822 constitue un point tournant dans la pensée de Viger, et la rhétorique de ses écrits subséquents s'en trouve profondément modifiée. En fait, Viger ne digère pas le projet d'union de 1822, projet qui émane de l'oligarchie coloniale britannique, dissimulé à la population canadienne et qui a failli être adopté par le Parlement impérial. Pour Viger, ce projet d'union constituait un " acte d'iniquité politique " ourdi par " les habiles combinaisons d'un petit nombre d'hommes restés étrangers au milieu de nous, qui n'étaient pas satisfaits de s'y trouver placés au premier rang et d'y jouir à peu près de tous les avantages attachés à une caste privilégiée ${ }^{73}$ ». Selon lui, le "but hautement avoué à cette époque [...] était d'ensevelir d'un coup dans une ruine commune toutes nos institutions ${ }^{74} »$. Viger est d'ailleurs l'un des plus ardents opposants au projet d'union, si bien que le mouvement antiunioniste - composé d'assemblées populaires, de pétitions, d'articles de journaux et de comités - prend le nom de vigerie $^{75}$.

Toutefois, Viger n'en veut pas à Londres pour le projet d'union, car il croit que c'est le fait d'une "faible minorité qui a eu assez d'influence ici pour paralyser à la fois les intentions du gouvernement lui-même, pour l'entraîner dans l'erreur dans plus d'une occasion ${ }^{76}$ ". En 1831, il remercie d'ailleurs chaleureusement les parlementaires britanniques qui ont fait avorter le projet : "Honneur et reconnaissance à ces hommes énergiques et éclairés dont la voix s'est fait entendre dans le parlement impérial ${ }^{77}$. „Viger croit donc en la bonne foi des hommes politiques londoniens à l'égard des Canadiens, mais il croit également que cette bonne foi est détournée par l'avidité et l'antipathie de la minorité coloniale du Bas-Canada.

\footnotetext{
73 Viger, Considérations, 1831, p. 51.

74 Ibid.

75 Ouellet et Lefort, « Denis-Benjamin Viger ».

76 Viger, Considérations, 1831, p. 26.

77 Ibid., p. 53.
} 
Dans la dernière partie des années 1820 , la tension monte entre la majorité canadienne à l'Assemblée et l'exécutif colonial. Le Canada Tenures Act de 1825, qui visait à favoriser la commutation volontaire de la tenure seigneuriale à la tenure libre et, également, à faciliter le passage du droit de propriété français au droit de propriété anglais, est perçu comme une agression contre le caractère français du BasCanada et les droits de la population canadienne ${ }^{78}$. De plus, dans les seigneuries les terres à distribuer aux habitants canadiens commencent à manquer. Ceux-ci doivent s'entasser dans l'enclave seigneuriale qui leur est concédée, alors que les townships regorgent de terres cultivables qui sont toutefois monopolisées par la Couronne, qui les distribue au clergé anglican, à des spéculateurs et autres amis de l'administration coloniale.

À l'Assemblée, la majorité canadienne voit l'administration de la colonie lui échapper progressivement et certains plans d'assimilation se concrétiser. Le 14 juillet 1827, dans un discours à Montréal durant la campagne électorale qui donnera une forte majorité canadienne à l'Assemblée, Viger exhorte les habitants à s'unir et à faire valoir leurs droits s'ils ne veulent pas perdre toute influence dans le gouvernement de leur pays, comme les Irlandais en Irlande, soumis au diktat d'une minorité étrangère ${ }^{79}$.

En 1828, en compagnie de John Neilson et d'Augustin Cuvillier, Viger est mandaté par l'Assemblée pour aller à Londres présenter les griefs de la Chambre contre le gouverneur Dalhousie. Croyant en la bonne foi de Londres, l'Assemblée espère que la métropole rendra justice aux Canadiens. On reproche notamment à l'administration coloniale les nominations aux Conseils législatif et exécutif alors qu'en 1828, seulement 9 des 27 conseillers sont nés au Bas-Canada et que les autres sont presque tous d'origine britannique. En outre, certains sont membres des deux Conseils. Viger établit un lien entre ces

${ }^{78}$ Voir Jean-Paul Bernard, Les rébellions de 1837 et de 1838 dans le Bas-Canada, Ottawa, La Société historique du Canada, brochure historique n ${ }^{\circ}$ 55, 1996, p. 23.

79 Lefort, Les deux missions de Denis-Benjamin Viger en Angleterre, p. 89. 
nominations non canadiennes et le fait que le Conseil législatif repousse la plupart des projets de loi de l'Assemblée qui visent à donner plus de pouvoirs aux représentants canadiens ou à mieux défendre les intérêts de la population canadienne. De même, devant le gaspillage d'argent et l'utilisation qu'en fait parfois l'oligarchie pour ses propres intérêts et pour payer les sinécures des coloniaux, un des griefs porte sur la gestion des finances. Par conséquent, l'Assemblée désire voter chaque année les crédits de façon détaillée tandis que le gouverneur et les conseillers tiennent à un vote global. Spécialiste du droit anglais, Viger cite d'ailleurs le célèbre juriste anglais William Blackstone en appui aux revendications de l'Assemblée. Ce dernier soutenait que « la Chambre des Communes a le privilège de prendre l'initiative d'accorder les crédits ${ }^{80}$ ". Ainsi, la députation canadienne revendique pour l'Assemblée au Bas-Canada les mêmes privilèges que les Communes à Londres. La mission est couronnée d'un certain succès puisque Londres rappelle Dalhousie et reconnaît à l'Assemblée le droit de voter les subsides. Cependant, malgré les tentatives de coopter certains Canadiens en les nommant au Conseil législatif, cette branche de la législature posera toujours problème.

C'est durant cette mission diplomatique de 1828 que Viger visite la France et les Pays-Bas et qu'il est témoin des motifs de mécontentement entre les Belges et les Hollandais. Il décèle plusieurs aspects similaires entre les revendications des Belges et celles des Canadiens, de même qu'entre l'union de ces deux peuples distincts et les projets d'union des Canadas planifiés par l'oligarchie coloniale britannique. La révolution belge de 1830 lui inspire donc l'écriture d'un ouvrage de 67 pages, ses Considérations relatives à la dernière révolution de la Belgique, qui paraît en 1831.

Examiner les événements survenus en Belgique " peut être un moyen d'instruction, une source de réflexions éminemment utiles au peuple du Bas-Canada comme à tous ceux qui prennent part à

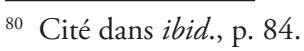


son gouvernement dans quelques degrés qu'ils se trouvent placés ${ }^{81}$ ". Par cet énoncé, on sent la rupture qui s'est opérée chez Viger. L'objectif n'est plus de persuader l'administration coloniale ou Londres, bien qu'il les invite à cogiter sur son ouvrage. On sent plutôt la volonté d'explorer de nouvelles avenues pour régler les problèmes des Canadiens. Viger laisse entendre que les événements de Belgique pourraient bien inspirer le Bas-Canada aux prises avec la domination de l'oligarchie coloniale.

L'ensemble de l'ouvrage porte sur l'analyse des motifs de discorde entre les Belges et les Hollandais et se termine par une comparaison entre les événements belges et les projets d'union des Canadas. C'est à la lumière du diagnostic que pose Viger sur les événements de Belgique qu'il confirme sa conception culturelle et holistique de la nation développée dans ses écrits de 1809 et de 1826. Pour lui, en effet, la révolution de la Belgique s'explique par les erreurs commises lors du Congrès de Vienne en 1815, qui a mené à l'annexion de la Belgique catholique et majoritairement francophone à la Hollande protestante et flamande. Lisons-le :

Les leçons de l'expérience sont trop souvent perdues pour ceux qui président aux destinées des nations. On trouve un exemple bien frappant de cet oubli du passé dans la conduite que l'on a tenue en Europe à la suite des évènemens qui avaient amené la chute de Bonaparte. Après avoir brisé le sceptre de la tyrannie qui avait pesé sur elle pendant tant d'années, on a servilement imité la conduite de l'ambitieux monarque qui avait disposé du sort des empires, abattu, relevé des trônes, établi, détruit, refait des constitutions et des gouvernemens, divisé, réuni des nations, morcelé des pays, amalgamé les peuples sans consulter les affections, les intérêts ou les besoins de ceux dont il réglait le sort ${ }^{82}$.

Cette longue citation, qui confirme la sensibilité conservatrice de Viger, exprime son adhésion au principe des nationalités voulant que les peuples doivent se gouverner eux-mêmes. Les différentes

81 Viger, Considérations, 1831, p. 2.

82 Ibid., p. 12. 
nations possèdent leur propre logique interne et leurs propres institutions culturelles. On ne peut jouer à Dieu avec les sociétés humaines, les manipuler, les amalgamer et tenter de les transformer, comme l'ont fait les monarques de la Sainte-Alliance lors du Congrès de Vienne, sans déranger l'ordre de ces nations et engendrer des troubles et des révolutions, comme celle survenue en Belgique. Ici, le lien que fait Viger entre cette union ratée et les projets d'union des Canadas est explicite.

L'imposition du néerlandais comme langue d'usage dans le domaine public, une représentation inégale avantageant les Hollandais, un système d'éducation et un système de justice dirigés par les Hollandais protestants sont autant de facteurs qui ont contribué à l'exaspération des Belges, suivant Viger, et au fait que ceux-ci se sont révoltés. Dans une fine analyse des sentiments qui guident les peuples à se révolter, Viger décrit le cheminement qui est le sien depuis 1809 et qui le conduira à radicaliser son discours à la veille des rébellions de 1837-1838 et à exiger des réformes démocratiques :

Les peuples sont rarement portés à troubler l'ordre qui les laisse dans la jouissance paisible de ce dont on ne peut tenter de les priver sans crime. L'idée de la résistance à l'autorité ne prend naissance chez eux qu'au moment où cet ordre lui-même éprouve quelque renversement. Ils souffrent avec résignation les abus du pouvoir autant de temps qu'ils conservent l'espoir de voir l'équilibre se rétablir. Ce n'est que lorsqu'ils n'attendent plus de justice de la part de ceux qui les gouvernent, qu'ils songent à s'agiter et à la demander comme une chose qui leur est dûe $[s i c]^{83}$.

On peut aussi comprendre ce passage comme un avertissement à mots couverts adressé aux autorités coloniales et britanniques sur ce qui risque de se produire si elles persistent à troubler l'ordre national canadien.

Chez le contre-révolutionnaire Joseph de Maistre, « [1] es nations ne s'aiment pas $^{84} »$. C'est pourquoi, selon lui, elles sont en conflit

${ }^{83}$ Ibid., p. 8-9.

${ }^{84}$ Cité dans Jacques Alibert, Joseph de Maistre : État et religion, Paris, Téqui, 1990, p. 119. 
perpétuel les unes contre les autres. Sans nécessairement partager cette vision ultrapessimiste, Viger n'en pense pas moins que l'union des deux Canadas ne serait pas possible, car elle réunirait deux nations aux mœurs, institutions et coutumes différentes et incompatibles, ce qui conduirait inéluctablement à l'éclatement et aux désordres, comme ce fut le cas en Belgique. Deux groupes nationaux distincts peuvent très bien s'entendre en se côtoyant, mais ils ne peuvent se fondre politiquement sans que l'un domine l'autre : «On donna la Belgique à la Hollande comme une ferme à exploiter. Dans le fait le sort de celle-ci fut de commander, l'autre fut de se soumettre et d'obéir ${ }^{85}$. " Viger constate, en citant de Maistre, que « la rage de domination étant innée dans l'homme [...] l'étranger qui vient chez une nation sujette au nom d'une souveraineté lointaine, au lieu de s'informer des idées nationales pour s'y conformer, ne semble trop souvent les étudier que pour les contrarier ${ }^{86} "$. Il est dans la nature de certains hommes d'agir ainsi, selon Viger. Son constat, en conclusion de son ouvrage, est sans équivoque : il fait sienne cette citation de Joseph de Maistre, qui exprime très bien l'esprit du principe des nationalités qui anime de plus en plus Viger : " $[\mathrm{N}]$ ulle nation ne veut obéir à une autre par la raison toute simple qu'aucune nation ne sait commander à une autre ${ }^{87}$. "

Yvan Lamonde et Marcel Bellavance ont bien souligné la présence d'un éveil nationalitaire dans le discours patriote, de même que l'adhésion au principe des nationalités chez plusieurs patriotes dans les années 1830. Ils ont cependant lié cette adhésion au principe des nationalités au libéralisme philosophique des Lumières ${ }^{88}$. Soulignons toutefois que l'éveil nationalitaire chez Viger ne se rattache pas à une conception libérale et individualiste de la société et de la nation, mais

${ }^{85}$ Viger, Considérations, 1831, p. 13.

${ }^{86}$ Ibid., p. 54.

${ }^{87}$ Ibid.

${ }^{88}$ Voir Lamonde, Histoire sociale des idées au Québec, p. 85-279; Marcel Bellavance, Le Québec au siècle des nationalités : essai d'histoire comparée, Montréal, VLB éditeur, 2004, p. 101-112. 
à la conception holistique et culturelle de la collectivité nationale issue du courant conservateur et romantique. Comme le remarque la sociologue Dominique Schnapper, deux interprétations principales ont été données du principe des nationalités : «On pouvait légitimer la revendication des peuples à disposer d'eux-mêmes au nom du peuple des citoyens ou au nom du peuple issu d'une histoire et d'une culture originelles $^{89}$. " Pour sa part, Viger appartient à la deuxième tendance, et c'est pour que le peuple canadien d'origine et de culture françaises puisse se gouverner lui-même qu'il en viendra à revendiquer des réformes démocratiques à partir de 1834 . Frustrées de ne pouvoir mener la colonie à leur guise, les élites marchandes britanniques veulent éliminer les institutions canadiennes qu'elles considèrent rétrogrades, car elles croient que les leurs sont supérieures et incarnent le progrès. Viger, pour sa part, est d'avis que les institutions canadiennes sont différentes et conformes au passé et au génie de la nation à qui elles appartiennent. Si des réformes doivent éventuellement advenir au sein des institutions canadiennes, celles-ci se feront d'ellesmêmes et émaneront de l'intérieur, c'est-à-dire de la dynamique interne de l'ordre canadien. Elles ne doivent pas être imposées de l'extérieur, c'est-à-dire à l'initiative impérialiste de l'oligarchie coloniale britannique.

Avec ses Considérations relatives à la dernière révolution de la Belgique, l'objectif de Viger était d'amener les Canadiens à méditer sur leur propre cas en s'inspirant des événements belges de 1830 . Viger soutient, en effet, qu' " en nous livrant à cet examen, nous trouverons aussi de nouveaux motifs de travailler même avec une ardeur nouvelle à réparer des erreurs dont nous souffrons ${ }^{90}$ ". Dans ses ouvrages subséquents, en 1834-1835, Viger semble avoir trouvé le moyen de réparer ces erreurs. Sa pensée nationaliste, qui s'est développée depuis le début du XIX ${ }^{\mathrm{e}}$ siècle, au gré des attaques hostiles de l'oligarchie au pouvoir dans la colonie, l'amène à considérer que

89 Dominique Schnapper, La communauté des citoyens, Paris, Folio, 2003, p. 231.

90 Viger, Considérations, 1831, p. 58. 
les Canadiens doivent se gouverner eux-mêmes en court-circuitant cette oligarchie et en réclamant que soient élus les membres du Conseil législatif. Ce sont ces revendications qu'il formule dans ses écrits de 1834 et 1835 .

\section{Le temps des revendications (1834-1837)}

En novembre 1829, peu de temps après son retour de Londres, Viger est nommé au Conseil législatif, poste qu'il accepte en espérant pouvoir contribuer à mettre fin au blocage du Conseil envers les projets de loi de l'Assemblée. Il y siège jusqu'à la suspension de la Constitution en 1837. Comme il le fera remarquer lui-même en 1834, l'objectif en le nommant au Conseil était d'attirer « quelques Canadiens influens, moins en vue de s'aider de leurs vertus et de leurs lumières, [...] que pour les arracher à la cause de leur pays en les noyant dans une majorité hostile ${ }^{11}$ ". Il ajoute l'année suivante que le but était de l'acheter en espérant que le fait d'être nommé conseiller flatterait suffisamment sa vanité et son ambition pour qu'il cesse de revendiquer des réformes, "comme si des intérêts de corps auraient dû l'emporter, dans le cœur d'un citoyen, sur celui de son pays et de son gouvernement ${ }^{92}$ ".

Toutefois, Viger ne siège pas très longtemps car, en mai 1831, il part à nouveau en mission à Londres, mandaté par la Chambre d'assemblée. Il ne revient au Bas-Canada qu'en novembre 1834. Le but de la mission est d'exposer les problèmes de la population canadienne et d'obtenir les changements nécessaires au système colonial $^{93}$. Entre 1827 et 1835 , huit secrétaires aux colonies et huit sous-secrétaires d'État se succèdent. Chacun a une politique différente

${ }^{91}$ Observations sur la réponse de Mathieu Lord Aylmer [...] et sur le discours du très honorable E. G. Stanley [...], Montréal, imprimé sous la direction d'un comité spécial du comité central et permanent du district de Montréal, 1834, p. 6.

92 Observations de l'Hon. D. B. Viger, contre la proposition faite dans le Conseil législatif le 4 de mars 1835 de rejeter le bill de l'Assemblée pour la nomination d'un agent de la province, Montréal, imprimé par Ludger Duvernay, 1835, p. 56.

93 Lefort, Les deux missions de Denis-Benjamin Viger en Angleterre, p. 382. 
et aucun n'a le temps de se familiariser suffisamment avec les affaires du Bas-Canada pour être en mesure de redresser la situation. Pour les chefs patriotes, ces changements fréquents au poste de ministre permettent aux bureaucrates coloniaux et au Conseil législatif de conserver la gouvernance de l'Empire. De plus, le Conseil protège les marchands britanniques à la tête de l'économie du Bas-Canada en bloquant les projets de loi de l'Assemblée. De 1822 à 1836, le Conseil bloque pas moins de 302 projets de loi présentés par l'Assemblée ${ }^{94}$. Lors de sa mission, Viger exige donc en priorité la réforme du Conseil législatif. Cependant, selon André Lefort, qui s'est penché sur les deux missions de Viger à Londres, ce dernier ne réclame pas que soit élu le Conseil législatif avant juin 1833, car il croit que le gouvernement britannique modifiera l'esprit de cette Chambre en nommant suffisamment de Canadiens pour que ces derniers puissent diriger la deuxième branche de la législature et défendre leurs intérêts nationaux ${ }^{95}$. Ces modifications ne viendront toutefois pas.

En 1834, alors qu'il est toujours à Londres, Viger s’aperçoit que sa mission sera un échec et qu'il faut procéder autrement que par des missions diplomatiques pour obtenir la réforme du Conseil législatif. Le gouverneur du Bas-Canada, lord Aylmer, de même que le secrétaire aux Colonies, lord Stanley, ont clairement exprimé leur refus d'acquiescer aux demandes de réformer ce Conseil. Tous deux refusent de nommer davantage de Canadiens au Conseil législatif, car cela irait à l'encontre des intérêts britanniques. De surcroît, dans un discours devant la Chambre des communes, en avril 1834, Stanley présente les institutions canadiennes et les Canadiens eux-mêmes comme la principale source des problèmes du Bas-Canada. Selon ce que rapporte Viger, Stanley dépeint le Haut-Canada comme un monde d'harmonie alors que le Bas-Canada vivrait dans le chaos,

\footnotetext{
${ }^{4}$ Lamonde, Histoire sociale des idées au Québec, p. 122.

95 Lefort, Les deux missions de Denis-Benjamin Viger en Angleterre, p. 336.
} 
malgré le fait que les deux Canadas aient la même constitution ${ }^{96}$. Le secrétaire aux Colonies imputait de la sorte les troubles du Bas-Canada au dysfonctionnement des institutions canadiennes. C'est pourquoi Stanley et Aylmer prétendent que l'union est la seule façon de rétablir l'harmonie au Canada ${ }^{97}$.

Ainsi, en juillet 1834, en réponse à ces propos d'Aylmer et de Stanley, Viger fait publier ses Observations, dans lesquelles il pourfend le gouverneur du Bas-Canada et le secrétaire aux Colonies. Ce texte d'une quarantaine de pages marque la radicalisation du discours de Viger, qui réclame ouvertement des réformes démocratiques et la destitution de ceux qu'ils qualifient " d'ennemis les plus invétérés du pays ${ }^{98}$ ». Si les 92 Résolutions ont été rédigées en l'absence de Viger, son texte de 1834 montre qu'il s'inscrit en conformité avec certaines des revendications patriotes, notamment l'électivité du Conseil législatif.

Tout d'abord, Viger réitère ce qu'il considère être les doléances de la population canadienne face aux propos de Stanley, qui, pour justifier l'abolition du système seigneurial, prétendait dans son discours que ce système était une des causes d'agitation de la population face aux seigneurs :

La tenure féodale et les lois qui la régissent ne sont qu'une infiniment faible partie des lois civiles que les Canadiens veulent conserver dans leur ensemble, avec leur importance politique dans le pays de leur naissance, leur religion, leurs lois, leur langue, leurs moeurs, avec les égards et le respect et la protection, pour ces propriétés sacrées, de la part du Gouvernement qui les attaque et les outrage dans les invectives passionnées de Mr. Stanley99.

Viger reste cohérent dans ses revendications nationalistes jusqu'à l'aube des rébellions. Ses revendications démocratiques ne se conjuguent donc pas avec la volonté d'instaurer des institutions plus

\footnotetext{
96 Viger, Observations, 1834, p. 12.

${ }^{77}$ Lefort, Les deux missions de Denis-Benjamin Viger en Angleterre, p. 336-337.

98 Viger, Observations, 1834, p. 23.

99 Ibid., p. 22-23.
} 
libérales visant à permettre la propriété privée du sol par l'abolition de la tenure seigneuriale, mais elles s'inscrivent dans la foulée de ses revendications nationalistes, qui, elles, visent à permettre à la nation canadienne de se gouverner elle-même, avec ses institutions et dans sa propre logique nationale. Fernand Ouellet a bien fait ressortir cette nécessité chez Viger, qui défendait le régime seigneurial pour des considérations nationalistes, en voulant préserver « le droit et la structure économique sur lesquels » repose la nation canadienne ${ }^{100}$. De même, les institutions seigneuriales représentent un bon moyen de contrer le capitalisme des spéculateurs reposant sur la propriété individuelle à l'anglaise. Ce capitalisme, comme l'a souligné LouisGeorges Harvey ${ }^{101}$, est apparenté chez Viger à la corruption des mœurs et à la décadence de l'oligarchie coloniale, cette catégorie d'individus qui se distingue " par l'intérêt personnel, la corruption, la recherche de terres ou d'emplois pour eux et leurs amis ${ }^{102}$ ". Délaisser le régime seigneurial pour le système de franc et commun soccage à l'anglaise équivaudrait donc à permettre à l'avidité de quelques-uns de monopoliser les terres par l'accumulation, de même qu'à contribuer à l'endettement et à la paupérisation des habitants. En 1833, Viger et Augustin-Norbert Morin, tous deux représentants de l'Assemblée à Londres, remirent un mémoire au gouvernement impérial dans lequel ils s'opposaient à " l'octroi d'une charte à une compagnie (la British American Land Company) pour l'achat des terres dans le Bas-Canada ${ }^{103}$ ». Pour Viger, le système de franc et commun soccage,

en resserrant dans un nombre de mains proportionnément petit toute la propriété foncière, la grève au delà de sa valeur intrinsèque du prix du monopole et il en résulte journellement les marchés

100 Fernand Ouellet, "L'abolition du régime seigneurial et l'idée de propriété ", dans Éléments d'histoire sociale du Bas-Canada, Montréal, Hurtubise HMH, 1972, p. 297-315.

101 Harvey, Le printemps de l'Amérique française, p. 42-43.

102 Viger, Observations, 1834, p. 30.

103 Cité dans Thomas Chapais, Cours d'histoire du Canada, tome IV, Québec, Librairie Garneau, 1923, p. 48. 
les plus ruineux pour les preneurs à bail. Dans les champs, dans les villes, d'immenses propriétés sont couvertes d'une population qui s'appauvrit tous les jours puisqu'elle vit sur son capital ${ }^{104}$.

En plus de s'insérer dans la logique du système national canadien qu'il cherche à défendre, les institutions seigneuriales, en permettant aux habitants un accès facile et peu coûteux à la terre, sont plus conformes aux valeurs morales de Viger, qui considère l'accumulation et la spéculation engendrées par le capitalisme marchand reposant sur la propriété individuelle comme de "l'agiotage ${ }^{105}$ ». Ainsi, pour des motifs moraux et nationalistes, Viger s'oppose au processus d'expropriation inhérent au système capitaliste, qui voit les terres cultivables monopolisées par quelques spéculateurs et qui crée par le fait même, en paupérisant les habitants, d'importantes masses de prolétaires réduites à travailler dans les villes industrielles. À ce processus achevé qu'il constatait dans ses études sur l'Irlande et la Grande-Bretagne, Viger préférait le système seigneurial issu du régime français dans lequel l'habitant était propriétaire de sa terre, et les rentes à taux fixes.

Le discours de Viger en 1834 est marqué par une rupture lorsqu' il juge qu'il est temps de passer de la parole aux actes pour faire advenir les réformes souhaitées. Normalement conciliant et modéré, Viger semble suffisamment vexé, ou semble concevoir un danger suffisamment grand pour la nation canadienne, pour croire que la radicalisation du discours et l'action sont les seuls moyens d'obtenir gain de cause. Sa confiance en la bonne foi des politiques londoniens semble avoir été ébranlée par le discours de Stanley et l'échec de sa mission diplomatique. Il lance ainsi un appel à ses compatriotes et met sur pied un plan d'action visant à faire pression sur Londres pour qu'elle consente à des réformes démocratiques. Il soutient qu'il faut, dans un premier temps, ouvrir « la voie des remontrances et des protestations les plus fortes et les plus unanimes, comme vous et vos représentans

104 Viger, Observations, 1834, p. 27.

105 Viger, Observations, 1835, p. 68. 
les faites en ce moment ». Dans un deuxième temps, Viger s'inspire de l'exemple des Bostonnais et conseille au peuple : " restreignez vos consommations de produits anglais le plus généralement que possible. Soyez fiers de vous habiller de la toison de vos troupeaux et de la dépouille de vos champs ${ }^{106}$. " Dans cet appel au peuple, il insiste également sur l'objectif recherché : "Votre détermination est prise d'insister toujours jusqu'à ce que vous l'ayez obtenue, sur l'extension du systême électif; votre arrêt de condamnation contre la constitution actuelle du Conseil Législatif est irrévocable, de votre part ${ }^{107}$. "

Cet appel au peuple fait ressortir les influences démocratiques américaines chez Viger. D'ailleurs, dès la première page de l'ouvrage, il fait référence aux paroles de Thomas Jefferson dans la Déclaration d'indépendance américaine lorsqu'il défend l'honnêteté des " représentans délégués par le peuple et pour le peuple ${ }^{108}$ ». Il affiche alors clairement ses influences américaines, comme l'a montré LouisGeorges Harvey pour l'ensemble du mouvement patriote ${ }^{109}$.

Il réitère sa volonté de voir adopter des réformes démocratiques dans un discours qu'il prononce devant le Conseil législatif, où il siège, en mars 1835. Ce discours est l'objet d'un ouvrage publié durant la même année ${ }^{110}$. Ce texte de 79 pages, écrit à la troisième personne du singulier, lui offre l'occasion de se défendre d'avoir représenté les doléances de l'Assemblée à Londres malgré le fait qu’il était conseiller législatif. Tout en reprenant l'essentiel des motifs de mécontentement des Canadiens envers l'oligarchie coloniale, Viger reformule son souhait que soient apportés des changements démocratiques.

À nouveau, il souligne que l'oligarchie britannique est à l'origine du problème au Bas-Canada et que l'extension du principe électif aura pour effet de le régler. Il cite la confiscation arbitraire des biens

\footnotetext{
106 Viger, Observations, 1834, p. 30-31.

107 Ibid., p. 31.

108 Ibid., p. 3.

109 Harvey, Le printemps de l'Amérique française.

110 Viger, Observations, 1835.
} 
du Séminaire de Montréal, l'expropriation de " trois cents familles censitaires de La Salle des terres que leurs travaux avaient fécondées, couvertes d'habitations et de culture ${ }^{111}$ ", le monopole de la propriété territoriale dans les townships, comme étant l'œuvre des " partisans de l'ascendant d'une classe particulière de citoyens sur la masse des habitans du pays ${ }^{112}$ ". Viger établit un lien entre ces façons de faire de l'oligarchie et l'Irlande, là où « au commencement du dernier siècle, il restait à peine aux descendans de la nation la vingt-deuxième partie des possessions territoriales de leurs ancêtres ${ }^{113}$ ». Remarquant qu'au Bas-Canada l'oligarchie coloniale emploie un procédé analogue à celui utilisé en Irlande, il conclut que « les mêmes causes devaient nécessairement entraîner ici des conséquences semblables, si nous ne nous empressions d'en arrêter la marche ». Il réfute donc les arguments des membres de l'oligarchie coloniale qui s'opposent à l'électivité du Conseil législatif et veulent conserver le statut spécial de ce Conseil en y maintenant sa composition britannique :

En soutenant la nécessité prétendue d'une représentation spéciale dans notre branche de la législature en faveur d'une classe distincte, en réclamant pour cette classe des priviléges [sic] particuliers, un pouvoir exclusif, n'était-ce pas, en d'autres termes, demander pour elle cet ascendant dont le résultat avait depuis des siècles été si fatal à l'Irlande, où, sur le sol qui l'avait vu naître, tout un peuple s'était trouvé sans patrie ${ }^{114}$ ?

Le texte de Viger se termine par la description de l'évolution de sa pensée depuis le début du XIX ${ }^{\mathrm{e}}$ siècle. Il réitère son admiration pour la constitution mixte britannique et l'Acte de 1791 en affirmant

qu'il avait consacré son temps, des veilles laborieuses à l'étude des principes de son gouvernement [et qu'il] avait pu distinguer les élémens de liberté dont il se composait, l'égalité des droits qu'il

111 Ibid., p. 43.

112 Ibid., p. 47.

113 Ibid., p. 40.

114 Ibid., p. 41-42. 
assurait aux citoyens, les moyens efficaces qu'il mettait entre leurs mains pour résister à l'abus de pouvoir ${ }^{115}$.

C'est la raison pour laquelle Viger aimait ce type de gouvernement et qu'il le concevait comme le plus susceptible de " faire le bonheur des sociétés ". C'est aussi pourquoi ses compatriotes et lui se sont considérés comme des sujets britanniques et qu'ils ont défendu leur gouvernement contre les États-Unis en 1812. Mais Viger, en 1835 , est un homme déçu qui se sent trompé : "le prix de leur attachement à la métropole, celui de leurs sacrifices, devait être la dégradation pour eux et leurs descendans. Ils voyaient une classe particulière réclamer au milieu d'eux; et sur eux, la puissance législative [...] sous prétexte de son origine britannique ${ }^{116}$. "C'est ainsi qu'il en vint à revendiquer des réformes démocratiques et qu’à "ses yeux les règles de conduite devaient être l'expression de l'opinion générale ", c'est-à-dire celle du peuple canadien. Il termine son discours sur cette note qui, bien qu'il ne les ait pas souhaités, comme nous le verrons plus loin, annonce les soulèvements de 1837-1838 : " la confiance des peuples [est] la vie d'un gouvernement. [...] Dès le moment où la justice cessait de guider l'exercice du pouvoir, tous les liens des obligations morales se détachaient, il se creusait un abîme sous les fondemens d'une société117."

Il faut noter que, bien qu'il s'inspire quelquefois de l'exemple américain pour justifier les réformes démocratiques qu'il préconise, Viger, contrairement à son cousin Papineau et à une certaine frange du Parti patriote ${ }^{118}$, ne revendique jamais la république ni la rupture du lien colonial avec l'Angleterre. D'ailleurs, il semble que l'électivité du Conseil législatif soit la seule réforme démocratique qui lui importe vraiment, car elle est la seule qu'il réclame ouvertement. Comme le souligne Yvan Lamonde, la composition du Conseil législatif constitue

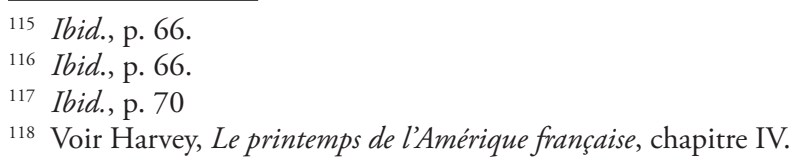


le "vice fondamental du système colonial " pour l'ensemble des patriotes ${ }^{119}$. Il semble que ce soit également le cas chez Viger. S'il fait référence une fois au gouvernement responsable qui prévaut aux États-Unis ${ }^{120}$, il ne revendique jamais ce type de gouvernement pour le Bas-Canada.

Il est constitutionnaliste et il croit en la Constitution mixte de 1791 - conforme à sa conception holistique, ordonnée et équilibrée de la société -, mais dans son optique, il faut que le pouvoir législatif en entier soit entre les mains des Canadiens afin qu'ils puissent se gouverner eux-mêmes, dans leur intérêt et en conformité avec leurs institutions et leurs mœurs. Pour lui, il faut donc que le principe aristocratique de la constitution du Bas-Canada, le Conseil législatif, soit électif pour permettre à des Canadiens de diriger cette branche de la législature. La citation qui suit exprime bien le constat de Viger selon lequel le problème du Bas-Canada n'est pas tant la Constitution de 1791 que l'oligarchie coloniale elle-même :

les abus dont il avait démontré les résultats obligés n'étaient pas moins le vice d'un système pernicieux que le fait des individus [...] En plaçant les hommes entre l'intérêt de la justice et celui de leurs passions, celles-ci devaient généralement faire pencher la balance en leur faveur [...] Cette représentation leur mettait entre les mains la puissance législative. Ils pourraient s'en servir d'une manière plus qu'indépendante de l'opinion publique, dans des vues différentes de celles du peuple du pays, dans des intérêts opposés ${ }^{121}$.

Lélectivité des conseillers législatifs a donc pour but de courtcircuiter l'oligarchie britannique hostile aux intérêts des Canadiens et de permettre ainsi à la Constitution d'être appliquée en conformité avec ses objectifs initiaux qui, selon Viger, étaient, " en nous en confiant le dépôt ", de "nous mettre à même de conserver nos loix,

\footnotetext{
119 Lamonde, Histoire sociale des idées au Québec, p. 122.

120 Viger, Observations, 1835, p. 32.

121 Ibid., p. 56-57.
} 
nos usages, notre langue, notre culte ${ }^{122}$ ». L'admiration de Viger pour le gouvernement mixte se subordonne donc à son nationalisme et c'est pourquoi il en vient à revendiquer l'électivité de la Chambre haute. Sa revendication démocratique va donc dans le sens du principe des nationalités, qui reconnaît le droit des peuples à disposer d'euxmêmes.

La possibilité pour le peuple canadien de se gouverner lui-même au sein d'un Bas-Canada sous protection britannique lui semble toutefois acceptable. C'est d'ailleurs l'appel qu'il fait à plus d'une reprise auprès des autorités coloniales : il suffit « de laisser la colonie à ellemême pour que le gouvernement Anglois n'eût rien à craindre des dispositions des habitans de la Province ${ }^{123}$ ". En ce sens, Viger n'est pas anticolonialiste. Néanmoins, cette acceptation du lien colonial ne signifie pas que Viger renonce à une éventuelle indépendance politique du Bas-Canada. Dès 1819, il écrivait : "Quand les circonstances amèneroient la séparation des deux pays, en supposant même que l'Angleterre crût devoir se déterminer à nous laisser jouir de l'indépendance politique $[\ldots]^{124}$. » À la lumière de cette citation, il y a lieu de croire que l'indépendance politique du Bas-Canada est une chose envisageable. D'ailleurs, dans son Histoire de la résistance, parue en 1839, Louis-Joseph Papineau rapporte que lord Bathurst, ministre des Colonies en 1823, lui aurait alors affirmé que lorsque la menace représentée par les États-Unis serait dissipée, « l’Angleterre serait prête alors à octroyer aux colonies qui leur seraient demeurées fidèles, et leur indépendance et des institutions meilleures que celles qui reposent sur le pacte fédératif ${ }^{125}$ ". Il est fort probable que Papineau ait fait mention de cet échange à son cousin et compagnon de lutte au sein du Parti canadien, puis patriote. En revanche, plus modéré et conservateur que son jeune cousin, Viger n'est pas anticolonialiste ou républicain dans

122 Viger, Analyse d'un entretien, p. 31.

${ }^{123}$ Ibid., p. 38.

124 Ibid., p. 39.

125 Louis-Joseph Papineau, Histoire de la résistance du Canada au gouvernement anglais, présentation de Georges Aubin, Montréal, Comeau et Nadeau, 2001 [1839], p. 16. 
les années 1830. S'il envisage sans doute l'indépendance politique de la nation canadienne à moyen ou à long terme, celle-ci se fera graduellement et s'imposera d'elle-même, lorsque les Canadiens formeront une nation suffisamment solide et imposante pour résister à la puissance militaire des États-Unis et à l'attrait qu'ils exercent.

Viger n'en est pas moins impliqué dans le mouvement revendicateur et réformiste jusqu'en 1837. Conformément au plan qu'il a présenté dans son ouvrage de 1834, l'objectif est de faire pression sur Londres. Il préside le banquet de la Saint-Jean-Baptiste à l'hôtel Rasco en juin 1835, de même que les banquets de 1836 et 1837. En 1835, il devient président de l'Union patriotique de Montréal. De même, la Banque du peuple, fondée par son cousin Louis-Michel Viger, émet des billets de 10 \$̀ son effigie. Ces faits montrent bien l'importance de Viger au sein du mouvement patriote dans les années 1830.

Néanmoins, le plan de Viger et des patriotes, qui consistait à faire pression sur le gouvernement de Londres, échoue totalement lorsque celui-ci répond aux 92 Résolutions par les Résolutions Russell, au printemps 1837. Non seulement Londres refuse de rendre le Conseil législatif électif et de répondre aux autres demandes contenues dans les 92 Résolutions, mais elle dépouille la Chambre d'assemblée de son droit de regard sur les dépenses de la colonie en permettant au Conseil exécutif d'outrepasser l'Assemblée. La position de Viger devant ce refus reste controversée.

Fernand Ouellet accorde de la crédibilité au témoignage de la servante de Viger, Angélique Labadie. Selon elle, son maître aurait eu des entretiens avec Papineau et aurait préconisé d'attendre la prise des glaces pour mener une insurrection armée ${ }^{126}$. Ce témoignage, livré dans un climat politique des plus troubles et angoissants, ne concorde toutefois pas avec les faits. Le 23 novembre 1837, Viger

126 Ouellet et Lefort, « Denis-Benjamin Viger ». Nous mentionnons uniquement le nom de Ouellet dans le texte puisque, dans sa thèse de doctorat, Lefort affirme qu'il ne croit pas en la crédibilité de ce témoignage (voir Lefort, Les deux missions de Denis-Benjamin Viger en Angleterre, p. 32-35). 
signe une adresse Aux habitans du district de Montréal avec treize autres magistrats, dans laquelle il invite ses concitoyens "non seulement à $\left[s^{\prime}\right]$ abstenir de toute démarche violente; mais encore à rentrer paisiblement dans [leurs] foyers ${ }^{127}$ ". De même, dans une correspondance avec John Arthur Roebuck en novembre 1838, Louis-Joseph Papineau soutient que Viger n'était sans doute pas lié au plan d'insurrection de 1838, car il ne croyait pas qu'une insurrection conduirait à la victoire des patriotes ${ }^{128}$. D'ailleurs, dans une lettre adressée à Louis-Joseph Papineau en août 1838, Viger écrit qu'il endossait les moyens de pression tant qu'ils se déroulaient dans les limites de la légalité, mais qu'il avait pris la décision de s'isoler à partir de l'automne de 1837, car ses principes lui interdisaient de prendre part à une révolte armée ${ }^{129}$. Viger est d'ailleurs absent lors de l'assemblée des Six Comtés à Saint-Charles en octobre.

Si Viger voulait faire pression sur Londres pour obtenir satisfaction, il n'allait pas jusqu'à souhaiter une insurrection armée. Néanmoins, celle-ci eut lieu et fut écrasée par les troupes britanniques. Les mandats d'arrêt émis contre les chefs patriotes par le gouverneur Gosford, le 16 novembre 1837, de même que la radicalisation militaire des milices et des volontaires loyaux ont précipité les événements qui ont conduit aux affrontements armés des mois de novembre et décembre. Dans un texte écrit en 1841, Viger accuse l'oligarchie britannique du Bas-Canada d'avoir précipité les événements pour inciter les Canadiens «sachant qu'ils n'étaient pas préparés, [...] à recourir aux armes, afin d'entraîner le pays dans la guerre civile, d'avoir un prétexte de déclarer la loi martiale et de réduire ainsi sans retour le pays sous le joug de leur odieux despotisme ${ }^{130}$ ». C'est à la

127 Le Populaire, 24 novembre 1837, p. 3, [En ligne], [http://collections.banq.qc.ca/ ark:/52327/1568938\#].

128 Louis-Joseph Papineau, Lettres à divers correspondants, t. $1: 1810-1845$, texte établi et annoté par Georges Aubin et Renée Blanchet, Montréal, Varia, 2006, p. 424.

129 Propos rapportés dans Lefort, Les deux missions de Denis-Benjamin Viger en Angleterre, p. 35.

130 L'Aurore des Canadas, 16 avril 1841, p. 2, [En ligne], [http://collections.banq. qc.ca/ark:/52327/1965321\#]. 
suite d'une seconde insurrection, en novembre 1838, après que la loi martiale eut été décrétée une seconde fois, que Viger est jeté en prison. Il y restera jusqu'en mai 1840. Lorsqu'il est libéré, l'Union des Canadas, depuis si longtemps planifiée et souhaitée par l'oligarchie coloniale et si farouchement combattue par Viger, est en voie d'être adoptée à Londres, avec l'objectif avoué d'assimiler la population canadienne.

\section{Conclusion}

Tant qu'un peuple n'est pas mort, il y a en lui, il y a dans sa religion et dans sa nationalité un principe d'énergie et de résurrection qu'un génie habile et fort peut féconder, remuer, régénérer et conduire à une glorieuse transformation.

Denis-Benjamin VigeR ${ }^{\text {I3I }}$

Dès ses premiers écrits au début du XIx ${ }^{\mathrm{e}}$ siècle, Denis-Benjamin Viger affiche une pensée nationaliste et conservatrice. Face aux bouleversements occasionnés par la situation coloniale des Canadiens, soumis à la domination d'une puissance étrangère, Viger défend la conservation, l'autonomie et la vitalité d'un peuple de souche, de langue et de culture françaises sur le territoire du Bas-Canada. Il use alors d'un discours conservateur inspiré des écrits d'Edmund Burke et de certains aspects de la pensée nationaliste de Joseph de Maistre et il développe une pensée systémique pour étayer ses arguments et défendre son propos. Pour Viger, les Canadiens, leur langue, leur culture, leurs mœurs et leurs institutions forment un système cohérent forgé par l'histoire, qu'il ne faut pas chercher à troubler ou à transformer, sans quoi le système entier risque de s'effondrer. Ainsi, ses premiers écrits visent, tout d'abord, à persuader les élites coloniales britanniques du bien-fondé de conserver intact et autonome le peuple canadien au sein du territoire du Bas-Canada. L'homme faisait partie de cette première cohorte de politiques canadiens issue

131 L'Aurore des Canadas, 15 août 1843, p. 3, [En ligne], [http://collections.banq.qc.ca/ ark:/52327/1965321\#]. 
des professions libérales et groupée autour du Parti canadien de Pierre Bédard, qui avait accueilli favorablement l'Acte constitutionnel et qui avait cru, à tort, que l'octroi d'un territoire séparé pour les Canadiens signifiait que Londres consentait à voir s'épanouir un peuple d'origine française sous sa protection. La citation qui ouvre notre article et qui affirme que l'Acte constitutionnel octroyait un territoire séparé aux Canadiens pour leur permettre « de conserver nos loix [sic], nos usages, notre langue, notre culte », est la démonstration la plus manifeste de l'adhésion de Viger à cette interprétation erronée de l'Acte de 1791. L'idée selon laquelle le peuple canadien pourrait s'épanouir et se fortifier sous la protection de l'Angleterre plaisait à Viger et lui fit perdre de vue que la métropole anglaise avait ses propres intérêts dans la possession de ses colonies.

Toutefois, le projet d'union des deux Canadas de 1822 et l'hostilité des élites coloniales britanniques envers les Canadiens lui firent comprendre la précarité et la vulnérabilité des siens. Il se mit alors à la recherche de remèdes. La révolution belge de 1830 et le courant nationalitaire européen fournirent ainsi d'excellents cas d'analyse à Viger, qui retint principalement cette citation de Joseph de Maistre, exprimant à merveille le principe des nationalités : « [N]ulle nation ne veut obéir à une autre par la raison toute simple qu'aucune nation ne sait commander à une autre. » Pour Viger, toutefois, l'affirmation nationalitaire ne se conjugue pas avec le libéralisme philosophique des Lumières, mais plutôt avec des idées conservatrices. S’il adhère au principe des nationalités, ce n'est pas pour instaurer des institutions libérales chez une masse d'individus citoyens, mais pour légitimer le droit du peuple canadien d'origine et de culture françaises d'être autonome, de conserver ses institutions nationales sur son territoire et de se gouverner lui-même, en dirigeant les deux Chambres législatives du gouvernement mixte et en court-circuitant ainsi l'oligarchie coloniale hostile au peuple canadien.

C'est en ce sens que, à l'instar des autres membres du Parti patriote, il revendique, de 1834 à 1837, l'électivité du Conseil législatif pour donner aux Canadiens la majorité à cette Chambre. Cependant, 
si Viger s'inspire quelquefois de l'exemple américain dans ses revendications, ce n'est pas parce qu'il adhère au républicanisme, mais pour légitimer la réforme démocratique qu'il exige. Il s'accommode bien du régime constitutionnel mixte britannique au sein duquel il observe un sain équilibre sociétal conforme à ses vues conservatrices et systémiques. Ainsi, jusqu'en 1837, Viger participe au mouvement patriote et aux moyens de pression visant à obtenir l'électivité du Conseil législatif. Cependant, l'échec de ces moyens de pression, le dérapage des événements et les soulèvements qui s'ensuivent incitent Londres à réaliser l'union des deux Canadas planifiée depuis le début du XIX ${ }^{e}$ siècle. 